\title{
Mittelalterliche Handschriften und Fragmente aus dem Stadtarchiv in Meißen Ergebnisse eines Erschließungsprojektes am Leipziger Handschriftenzentrum"
}

\author{
von \\ MATTHIAS EIFLER und WERNER J. HOFFMANN
}

Im Zuge zweier von der Deutschen Forschungsgemeinschaft geförderter Projekte werden am Leipziger Handschriftenzentrum ${ }^{1}$ seit 2010 ,Kleinsammlungen mittelalterlicher Handschriften und Fragmente in Ostdeutschland erschlossen. ${ }^{2}$ Es handelt sich um Bestände, die in verschiedenen Einrichtungen (Bibliotheken, kirchlichen und städtischen Archiven sowie Museen) aufbewahrt werden und der Forschung bislang weitgehend unbekannt waren. Im Zuge ihrer Erschließung kam es zu zahlreichen Neufunden und Entdeckungen.

$\mathrm{Zu}$ den bearbeiteten Beständen gehören auch sechs Handschriftenbände sowie 42 Handschriftenfragmente, die heute im Stadtarchiv Meißen aufbewahrt werden. Bei fünf der sechs Codices handelt es sich um Bände, die ehemals aus der Bibliothek der Fürsten- und Landesschule St. Afra in Meißen stammen und bei deren Auflösung 1948 in das Stadtarchiv Meißen verbracht wurden. Die Fragmente wurden, wahrscheinlich um 1945 oder 1946, von Akten des Stadtarchivs abgelöst oder kamen auf anderem Weg in das Archiv. Außer einer Sammelhandschrift (Signatur: H 3), die der bekannte Leipziger Gelehrte Johannes Fabri von Donauwörth $(† 1505)$ während seines Artes-Studiums (1472-1476) angelegt hatte, ${ }^{3}$ waren

* Der vorliegende Aufsatz ist ein gemeinschaftliches Werk, für Kapitel I bis III zeichnet als Autor Matthias Eifler, für Kapitel IV Werner J. Hoffmann verantwortlich. - Der letzte Zugriff auf alle im Folgenden genannten Internetquellen erfolgte am 8. August 2018.

1 Weitere Informationen zum Leipziger Handschriftenzentrum und zu den dort durchgeführten Projekten unter: https://www.ub.uni-leipzig.de/forschungsbibliothek/hand schriftenzentrum/. - Dem Leiter des Handschriftenzentrums Dr. Christoph Mackert ist für die Lektüre des Manuskripts dieses Aufsatzes sowie alle Hinweise und Anregungen sehr herzlich zu danken.

2 Informationen zu beiden Projekten, Handschriftenbeschreibungen (teilweise mit Digitalisaten) sowie Verweise auf weiterführende Literatur finden sich auf den Projektseiten: http://www.manuscripta-mediaevalia.de/info/projectinfo/leipzig-klein.html sowie http://www.manuscripta-mediaevalia.de/info/projectinfo/kleinsammlungen_ ostdeutschland.html.

3 Vgl. zu dieser Handschrift: KarL Manitius, Eine Sammelhandschrift des 15. Jahrhunderts aus der ehemaligen Fürstenschule St. Afra in Meißen, in: Forschungen und Fortschritte. Nachrichtenblatt der deutschen Wissenschaft und Technik 36 (1962), 
diese Manuskripte der Forschung bislang unbekannt. Da sie verschiedene Bezüge zur Stadt- und Schulgeschichte Meißens aufweisen, sollen im Folgenden einige Ergebnisse des Projektes vorgestellt werden.

\section{Die Sammelhandschrift des Görlitzer und Meißner Predigers Donatus Cluge (H S)}

Bei der Handschrift mit der Signatur H $5^{4}$ handelt es sich um einen 381 Blätter umfassenden Codex im Folioformat $(31 \times 21 \mathrm{~cm})$, der Texte zur Predigtvorbereitung enthält, nämlich als Haupttext Teil 1 der vom Franziskaner Johannes Erfordensis verfassten, alphabetisch geordneten Enzyklopädie biblischer Namen und Begriffe Tabula originalium ${ }^{5}$ (Abb. 1) sowie verschiedene kleinere Texte. Alle Texte wurden von einem Schreiber angelegt, der wohl auch die Ausstattung mit roten Überschriften und einfachem Buchschmuck übernahm. An zwei Stellen nennt der Schreiber seinen Namen, so auf dem vorderen Spiegel, wo er eine 1472 niedergeschriebene Liste der Meißner Bischöfe (Abb. 2) mit dem Schreibereintrag $>$ Per me Donatum Cluge est conscriptum< versah, sowie am Ende eines nachgetragenen Textes, dessen Schreiberkolophon lautet: [...] finitus per me Donatum 1479 feria quinta ante Natalis domini [20. Dezember 1479] in Misna (Bl. 378v). Daraus lässt sich schließen, dass in der komplett von Donatus Cluge geschriebenen Handschrift zumindest die Nachträge 1472 und 1479 in Meißen eingetragen wurden. Die Wasserzeichen 6 belegen aber, dass der Hauptteil des Bandes bereits um 1460/62 geschrieben worden und daher von verschiedenen Produktionsphasen auszugehen ist.

Donatus Cluge de Roßrwyn (Roßwein, ca. 30 Kilometer südwestlich von Meißen) wurde im Wintersemester 1442 an der Leipziger Universität immatrikuliert und im Sommersemester 1444 Baccalaureus sowie im Wintersemester 1451 Magister artium.7 Er wurde am 22. Juli 1453 zum Cursor und am 22. August 1460

S. 313-317; sowie zur Biografie und zum Werk des Johannes Fabri: Jürgen Stohlmann, Johannes Fabri, in: Kurt Ruh u. a. (Hg.), Die deutsche Literatur des Mittelalters. Verfasserlexikon, 2. völlig neu bearbeitete Auflage, 14 Bde., Berlin/New York 19782008 (im Folgenden: Verfasserlexikon), hier Bd. 2 (1980), Sp. 691-698. Die Neubearbeitung hat auch bei dieser Handschrift, v. a. im kodikologischen Bereich, zu neuen Erkenntnissen geführt; vgl. die ausführliche Beschreibung unter: http://www. manuscripta-mediaevalia.de/dokumente/html/obj31588399.

4 Vgl. die ausführliche Beschreibung: http://www.manuscripta-mediaevalia.de/doku mente/html/obj31586457.

5 Zum Autor (Lektor am Ordensstudium der Franziskaner, seit 1275 in Erfurt, seit ca. 1285-1295 in Magdeburg, nach 1295 erneut in Erfurt tätig) sowie zum Werk vgl. Norbert Brieskorn/VOLKer Honemann, Johannes von Erfurt, in: Verfasserlexikon (wie Anm. 3), Bd. 4 (1983), Sp. 583-589, v. a. Sp. 587 f.

6 Nachweise in der Beschreibung; siehe Anm. 4.

7 Vgl. Georg Erler (Hg.), Die Matrikel der Universität Leipzig (Codex Diplomaticus Saxoniae Regiae II/16-18), 3 Bde., Leipzig 1895-1902, hier Bd. I, S. 142, Bd. II, S. 134, 153. 
zum Sententiarius graduiert, ${ }^{8}$ scheint anschließend aber nicht zum Doktor der Theologie promoviert worden zu sein. Letztmalig wird er im Wintersemester 1467 an der Leipziger Universität erwähnt. ${ }^{9}$ Zwar schweigen die universitären Quellen über seinen anschließenden Lebensweg, Nachrichten über weitere Lebensstationen lassen sich aber durch die Kolophone in Büchern aus seinem Besitz ermitteln. Bislang konnten außer der Meißner vier weitere Handschriften in Görlitz, Wrocław und Prag aufgefunden werden, die vollständig von Donatus Cluge angelegt wurden. ${ }^{10}$ Mindestens vier weitere Codices waren in seinem Besitz, zwei davon stiftete er dem Zisterzienserkloster Altzelle. ${ }^{11}$ Aus den Schreiberkolophonen geht hervor, dass

8 Vgl. ebd. Bd. II, S. 9.

9 Vgl. ebd., S. 207, hier gemeinsam mit Stanislaus Pechmann de Schweidnitz als clavigeri magistri. Den clavigeri (Schlüsselträgern oder -verwaltern) oblag die Verwaltung eines der Schlüssel der Fakultätskasse.

10 Görlitz, Oberlausitzische Bibliothek der Wissenschaften (im Folgenden: OLB), Depositum der Görlitzer Innenstadtgemeinde, ohne Signatur (Biblia mit Glossa ordinaria, geschrieben 1467/68 in Leipzig, ausgestattet wohl in der Werkstatt des „Illuminators der Tulinkhoff-Bibel“, gebunden in der Leipziger Werkstatt Fritz Forchem [Friedrich Pflugritter aus Forchheim], später im Besitz der St. Peter- und Paulskirche Görlitz, 2007 von der Görlitzer Innenstadtgemeinde zurückgekauft, freundliche Mitteilung von Matthias Wenzel, OLB Görlitz, vom 20. Oktober 2016). - Praha, Národní knihovna České republiky (Nationalbibliothek, im Folgenden: NB), IV.D.1 (Gregorius Magnus: Homiliae quadraginta, Expositio super Cantica canticorum, Dialogi, etc., Testamentum et regula b. Francisci, geschrieben 1455 bis 1465 in Görlitz); vgl. JosepH TruHLÁr, Catalogus codicum manu scriptorum latinorum, qui in c. r. bibliotheca publica atque Universitatis Pragensis asservantur, Prag 1905, Bd. 1, Nr. 655, S. 262 f.; Beschreibung und Digitalisat: http://www.manuscriptorium.com/apps/index.php?direct=record\& pid=AIPDIG-NKCR_IV_D_1__OU6JZWB-cs. - Wrocław, Biblioteka Universytecka (Universitätsbibliothek, im Folgenden: UB; ehemals Görlitz, OLB), Mil. II 24 (Johannes de Erfordia OFM: Tabula originalium, pars II, beendet 1463, Bibelkommentare, Ambrosius Autpertus: De conflictu vitiorum et virtutum, beendet 1455 in Leipzig, Sermones etc.); vgl. StanisŁaw Kadzielski/Wojciech Mrozowicz, Catalogus codicum medii aevi manuscriptorum qui in Bibliotheca Universitatis Wratislaviensis asservantur signa 6055-6124 comprehendens (Codices Milichiani, vol. 1), Wrocław 1998, Nr. 6077, Digitalisat (Mikrofilm): http://dk.bu.uni.wroc.pl/cymelia/display DocumentFotos.htm?docId=8200039031. - Wrocław, UB (ehemals Görlitz, OLB), Mil. II 69 (Johannes Versor: Quaestiones super libros Ethicorum Aristotelis, Aristoteles: Ethica, beendet 1450 in Leipzig, Boethius, Consolatio philosophiae, beendet 1445, Ps.Boethius, De disciplina scholarium, beendet 1447 in Chemnitz, Quaestiones super librum de anima Aristotelis, beendet 1440 in Leipzig etc.); vgl. ebd., Nr. 6121, Digitalisat (Mikrofilm): http://dk.bu.uni.wroc.pl/cymelia/displayDocumentFotos.htm?docId= 5002000340.

11 Wrocław, UB (ehemals Görlitz, OLB), Mil. II 3 und Mil. II 14 (Predigtsammlung, Sommer- und Winterteil), 1458 in Leipzig von Conradus Textoris de Bamberg geschrieben; vgl. KaDZielsKi/Mrozowicz, Catalogus (wie Anm. 10), Nr. 6057 und 6068; vgl. Digitalisat (Mikrofilm): http://dk.bu.uni.wroc.pl/cymelia/displayDocumentFotos. htm?docId=5002000336 (Mil II 3). Der Abtei Altzelle stiftete Cluge folgende Handschriften: Leipzig, Universitätsbibliothek (im Folgenden: UB), Ms 195 (Origines: Homiliae in Genesin et Exodum nach Rufinus, 12. Jahrhundert [1163]; vgl. Eintrag $2^{\mathrm{v}} / 3^{\mathrm{r}}:$ Liber veteris celle S. Marie collatus per magistrum Donatum Cluge); Ms 457 (Liber Sententiarum, Hugo Ripelin: Compendium theologicae veritatis etc., Ende 13. Jahrhundert; vgl. Eintrag 
Donatus Cluge in den Jahren 1463 bis 1465 als Prediger einer Pfarrkirche in Görlitz tätig war. ${ }^{12}$ Hier schrieb er auch Teil 2 der Tabula originalium (Buchstaben M-Z), der am 19. August 1463 vollendet wurde. ${ }^{13}$ Dies zeigt, dass der heute in Meißen befindliche Teil 1 der Tabula (Buchstaben A-L) vor 1463 und ebenfalls in Görlitz angelegt worden sein muss, was durch die Wasserzeichenbefunde bestätigt wird. In Görlitz erfolgte sicher auch die Bindung der beiden Tabula-Bände sowie anderer Handschriften aus Cluges Besitz. ${ }^{14}$

Spätestens im Sommer 1470 befand sich Donatus Cluge in Meißen. ${ }^{15}$ Im Jahr 1472 trug er auf dem vorderen Spiegel der heute in Meißen aufbewahrten Handschrift die bereits erwähnte Liste zu den Meißner Bischöfen ein (Abb. 2). Sie reichte zunächst bis zum 38. Bischof, Dietrich III. von Schönberg (1463-1476), später ergänzte der Schreiber den Namen von dessen Nachfolger Johann V. von Weißenbach (1476-1487). ${ }^{16}$ Die Angaben zur Amtszeit einzelner Bischöfe (z. B. zur Gründung von Stiften und Klöstern, zu Käufen und Verkäufen von Gütern sowie zur Einführung von Vikarien und Heiligenfesten $)^{17}$ zeigen, dass der Schreiber die

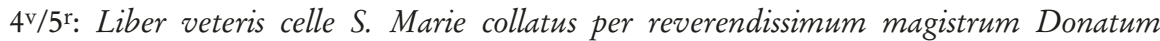
Cluge). Vgl. Die lateinischen und deutschen Handschriften der Universitäts-Bibliothek zu Leipzig, Bd. 1: Die theologischen Handschriften, beschrieben von Rudolf Helssig, Leipzig 1926, ND Wiesbaden 1995 (Katalog der Handschriften der Universitäts-Bibliothek Leipzig, Abteilung IV, Bd. 1), S. 275 f., S. 738-740.

12 Vgl. Mil. II 24 (vgl. Anm. 10), 385'r : Finitum per Donatum Cluge de Rossweyn sub anno domini millesimo quadringentesimosexagesimo tercio, feria 6 a post festum Assumpcionis Marie [19. August 1463] in parrachia Ga ${ }^{\mathrm{e}}$ rliczensi; Prag, NB, IV.D.1, 331v: [...] conscripta per me Donatum in $\mathrm{Ga}^{\mathrm{e}} \mathrm{rlicz}$ protunc ibidem pre[dicator?, Eintrag beschnitten], 409v:

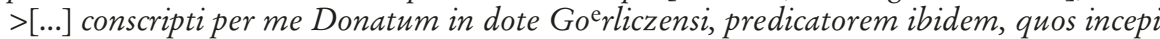
sub anno domini m ccc lxiiii [Wort zerstört] ebdomada proxima post festum Assumpcionis Marie, notabiliter vigente mortalitate hominum in idem et finiui in crastino sancte Katherine [26. November 1464], iam quammodo sedata dicta mortalitate [...], 278 $>$ Finitus est presens opus sub anno domini 1465 in die Ciriaci sancti martiris [8. August 1465] per me Donatum in $G a^{\mathrm{e}}$ rlicz). Die Bezeichnung in parrachia $\mathrm{Ga}^{\mathrm{e}}$ rliczensi bezieht sich wahrscheinlich auf eine der beiden Görlitzer Pfarrkirchen (St. Peter und Paul oder St. Nikolai); vgl. die Belege zur Verwendung des Begriffs ,Parrochialkirche' bei Christian Speer, Frömmigkeit und Politik. Städtische Eliten in Görlitz zwischen 1300 und 1550 (Hallische Beiträge zur Geschichte des Mittelalters und der Frühen Neuzeit 8), Berlin 2011, hier S. 63-65, v. a. Anm. 24.

13 Wrocław, UB, Mil. II 24 (vgl. Anm. 10).

14 Der Meißner Band wurde in der Werkstatt,Görlitz, Wappenmeister' gebunden. Auch die vom selben Schreiber angelegten Handschriften Wrocław, UB, Mil. II 24 und Prag, NB, IV.D.1 (vgl. Anm. 10) wurden in dieser Werkstatt gebunden. Einzelnachweise in der Beschreibung (vgl. Anm. 4).

15 Er ergänzte hier im Sommerteil der zweibändigen, 1458 in Leipzig geschriebenen und 1466 von ihm gekauften Predigtsammlung die letzte Predigt (vgl. Mil. II 3, 324vb: Per me Donatum in Mysna, 1470 feria 3 ante Corporis Christi [19. Juli 1470]); vgl. Anm. 11.

16 Eine zum Teil übereinstimmende, jedoch nur bis Johannes Hoffmann (1427-1451) reichende Liste findet sich auf dem hinteren Spiegel der Handschrift Leipzig, UB, Ms 1387; vgl. die Beschreibung unter: http://www.manuscripta-mediaevalia.de/doku mente/html/obj31577690.

17 Erwähnt werden u. a. die Gründung des Kollegiatstifts Wurzen durch Bischof Herwig (1106-1119) im Jahr 1114, des Kollegiatstifts Bautzen durch Bruno II. (1209-1228) 
Verhältnisse im Hochstift gut kannte oder eine entsprechende Vorlage benutzen konnte. 1479 war Cluge noch in Meißen und ergänzte am 20. Dezember den Text auf Bl. 373v $-378^{v}$, einen Ausschnitt aus dem Traktat über die Freundschaft von dem Grammatik- und Rhetoriklehrer an den Universitäten von Bologna und Padua Boncompagno da Signa ( $†$ nach 1240), ${ }^{18}$ später die zwei folgenden kurzen Texte, nämlich ein Formular für die Degradation eines Klerikers (Bl. 379r) sowie eine Ars memorativa (Bl. 379v-381v). ${ }^{19}$ Es ist also anzunehmen, dass Cluge von mindestens 1470 bis 1479 als Prediger in Meißen wirkte; für das Jahr 1472 gibt es dafür auch einen schriftlichen Beleg. ${ }^{20}$ Sehr wahrscheinlich war Cluge Inhaber des „Predigtstuhlamtes“ in der Meißner Domkirche, das 1419 von zwei Domherren eingerichtet worden war. ${ }^{21}$

Die Meißner Bischofsliste war wohl der Anlass dafür, dass die Handschrift 1906 aus der Auktion des Leipziger Antiquariats Karl W. Hiersemann für Meißen erworben worden ist, wobei die näheren Umstände leider unbekannt sind.22

sowie des Klosters St. Marienstern durch den späteren Bischof Bernhard von Kamenz (1293-1296) im Jahr 1248. Verzeichnet sind die Überführung der Reliquien des Hl. Donatus unter Bruno I. (1152-1154), die Einrichtung einer Vikarie zum Hl. Wenzel durch Johann II. von Jenstein (1375-1379), die Einführung des Festes des Hl. Viktor durch Nikolaus I. (1379-1392) sowie des Festes der Hl. Hedwig und der Inventio pueri Jesu durch Johannes Hoffmann (1427-1451).

18 Es handelt sich um einen Ausschnitt (Kap. 9-38) aus dem Traktat ,De amicitia“ des Boncompagno da Signa. Vgl. Sarin Nathan, Amicitia di Maestro Boncompagno da Signa (Miscellanea di letteratura del medio evo 3), Rom 1909, S. 46-88, hier S. 52-88. Bislang waren nur sechs Textzeugen (in Mailand, Paris, Rom, Berlin sowie Brüssel) bekannt; vgl. ebd., S. 42 f.

19 Einzelnachweise zu den Texten und ihrer Überlieferung in der Beschreibung (vgl. Anm. 4).

20 Dass Cluge 1472 praedicator ecclesiae Misnensis war, geht aus einem als Abschrift in einer Altzeller Handschrift (Leipzig, UB, Ms 1327, 413 v) enthaltenen Brief an den Pleban von Roßwein hervor, dessen Schlussformel lautet: Scriptus ex Misna feria sexta ante Palmarum [...] per vestrum Donatum Cluge Predicatorem Ecclesie Misnensis. Anno etc. $L X X I I^{\circ}$ [20. März 1472].

21 Das „Predigtstuhlamt“ in der Domkirche wurde 1419 von zwei Domherren (dem ehemaligen Dekan Johannes Poczte und dem ehemaligen Archidiakon Nicolaus Czoboth) testamentarisch eingesetzt; vgl. ERNST GOTTHELF GERSDORF (Hg.), Urkundenbuch des Hochstifts Meissen, Bd. 2 (Codex Diplomaticus Saxoniae Regiae II/2), Leipzig 1865, Nr. 900, S. 442-444. Der Amtsinhaber sollte ein praedicator notus et approbatus sein und an Sonn- und Feiertagen deutsch predigen (diebus dominicis et festivis ad beneplacitum decani et capituli sermonem ad vulgus seu in vulgari facere tenebitur). Der Anwärter sollte ein Graduierter der Theologie oder des kanonischen Rechts oder zumindest ein Baccalarius in einer der beiden Fakultäten sein; seine Wahl durch das Domkapitel sollte einstimmig erfolgen. Der Praedicator war zu dauernder Residenz verpflichtet und durfte nur bei äußerst zwingenden Gründen mit Erlaubnis des Dekans und Kapitels von Meißen abwesend sein.

22 Vgl. Auktionskatalog Karl W. Hiersemann, Katalog 330: Manuscripte des Mittelalters und späterer Zeit [...], Leipzig 1906, Nr. 7, S. 9 f. mit Angaben zur Handschrift, die als „Sehr interessantes Manuskript, von ganz besonderer Wichtigkeit für die Geschichte des Bistums Meissen“ bezeichnet wird. Sehr wahrscheinlich erfolgte der Ankauf auf Initiative des Meißner Geschichtsvereins oder einer Privatperson. Ob die Handschrift 
Wie die Handschriften des Donatus Cluge in Görlitz, Leipzig, Prag und Wrocław legt auch der Meißner Band ein bemerkenswertes Zeugnis von einem offenbar berühmten und mindestens zehn Jahre in Meißen wirkenden Domprediger ab, der in den archivalischen Quellen (also z. B. in Urkunden) aber überhaupt nicht zu fassen ist. Cluges Bände zeigen überdies sehr gut, welche Texte ein gelehrter Prediger an einer Domkirche zur Vorbereitung seiner Predigten benutzte.

\section{Mittelalterliche Handschriften aus der Bibliothek der Fürsten- und Landesschule St. Afra}

Bei fünf mittelalterlichen Handschriften des Stadtarchivs (Signatur H 1-4, H 6) zeigen bibliothekarische Einträge (etwa Rötelsignatur und Stempel der Bibliotheca Afrana), dass sie ursprünglich zur Bibliothek der 1543 gegründeten Fürsten- und Landesschule St. Afra in Meißen gehörten. Die Schulbibliothek von St. Afra war bis zum Jahr 1887 auf einen Bestand von 14500 Bänden angewachsen, ${ }^{23}$ darunter Bücher aus verschiedenen Stiftungen. Am Ende des Zweiten Weltkriegs erlitt die Sammlung starke Verluste und Schäden: Von den einstmals vorhandenen ca. 24000 Bänden blieben nach Kriegsende nur ca. 13000 erhalten. ${ }^{24}$ Da die Gebäude von St. Afra nicht weiter als Gymnasium, sondern als landwirtschaftliche Fachhochschule genutzt werden sollten, wurden im März 1948 umfangreiche Buchbestände in die Landesbibliothek nach Dresden verbracht, andere gelangten in verschiedene Institute der Universität Leipzig. ${ }^{25}$ Sehr wahrscheinlich erfolgte zu diesem Zeitpunkt auch die Übergabe der fünf mittelalterlichen Handschriften an das Meißner Stadtarchiv.

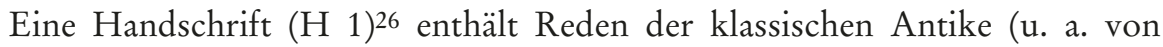
Sallust und Cicero) und wurde im letzten Viertel des 15. Jahrhunderts in Oberitalien oder der Toskana geschrieben. Die qualitätvolle Buchmalerei der Zierseite am Beginn (Abb. 3) legt eine Entstehung um 1480/90 in Florenz, evtl. im Umkreis des Buchmalers Attavante degli Attavanti († um 1525) nahe, der seit 1476 für die Medici, den Dom von Florenz, Herzog Federico von Urbino, aber auch für den ungarischen König Matthias Corvinus arbeitete. ${ }^{27}$ Wie ein querständiger Besitzein-

1906 bereits für das Stadtarchiv gekauft oder diesem erst später übergeben wurde, ist nicht bekannt.

23 Vgl. Beate Brück, Die Geschichte der Bibliothek der Fürsten- und Landesschule St. Afra zu Meißen von ihren Anfängen bis zu ihrer Auflösung im Jahre 1948, Diplomarbeit, Hochschule für Technik, Wirtschaft und Kultur Leipzig, Meißen 1992 [masch.], hier S. 33.

24 Vgl. ebd., S. 68.

25 Vgl. ebd., S. 60-65, hier S. 63 auch die fünf mittelalterlichen Handschriften erwähnt, die in das Meißner Stadtarchiv gelangten.

26 Ausführliche Beschreibung der Handschrift: http://www.manuscripta-mediaevalia.de/ dokumente/html/obj31587554.

27 Für Hinweise zur stilistischen Einordnung der Buchmalerei danke ich Frau Dr. Ulrike Bauer-Eberhardt, München, sehr herzlich (schriftliche Mitteilung vom 15. November 2016). 
trag28 (Abb. 3) zeigt, war der Band um 1702 in Prag und anschließend im Besitz des Diplomaten Heinrich van Huyssen (1666-1739). ${ }^{29}$ Dieser hatte u. a. in Duisburg, Leipzig und Wien Rechtswissenschaften, Geschichte und Rhetorik sowie in Köln und Halle alte Sprachen studiert, dürfte sich also für den Inhalt dieser rhetorischen Sammelhandschrift interessiert haben; 1689 wurde er in Straßburg zum Doktor beider Rechte promoviert. Van Huyssen war ein umtriebiger Reisender: Er bereiste mehrfach Italien und interessierte sich v. a. für die italienischen Bibliotheken und ihre Bücherschätze, ${ }^{30}$ war aber auch Prinzenerzieher, Diplomat und Historiograf am Hof Zar Peters I. Als Sekretär des sächsischen Generals Jakob Heinrich von Flemming reiste van Huyssen 1702 auch nach Prag, ${ }^{31}$ könnte die Handschrift dort also käuflich erworben haben. Nach van Huyssens Tod gelangte der Codex (vielleicht durch einen Verwandten) zunächst in die Universitätsbibliothek Wittenberg, wo er 1838 nachweisbar ist. ${ }^{32}$ Bald nach 1854 wurde die Handschrift dann vom Wittenberger Predigerseminar an den Oberlehrer und Bibliothekar am Gymnasium in Plauen Heinrich Theodor Flathe (1827-1900) abgegeben. ${ }^{33}$ Durch Flathe, der seit 1867 Professor und Bibliothekar der Landesschule St. Afra in Meißen war, gelangte sie in die Bibliotheca Afrana, wobei die genaueren Umstände unbekannt sind.

28 Auf $1^{\mathrm{r}}$ querständiger Besitzeintrag: Liber Manuscriptus [ein auf -tus (conventus?) endendes Wort radiert und ersetzt durch: H.buyssensis] Pragae Ao. 1702.

29 Zur Biografie vgl. u. a. Svetlana Korzun, Heinrich von Huyssen (1666-1739). Prinzenerzieher, Diplomat und Publizist in den Diensten Zar Peters I. des Großen (Jabloniana - Quellen und Forschungen zur europäischen Kulturgeschichte der Frühen Neuzeit 3), Wiesbaden 2013, v. a. S. 19-32.

30 Heinrich von Huyssen unternahm um 1690/91 eine Italienreise und verfasste darüber einen Reisebricht: Curieuse und vollständige Reiß-Beschreibung von gantz Italien [...], Freyburg [= Leipzig] 1701, Digitalisat: http://digi.ub.uni-heidelberg.de/diglit/huyssen 1701/0107. Darin berichtet er u. a. über den Besuch italienischer Bibliotheken (u. a. in Florenz, Rom und Venedig) und die dort eingesehenen mittelalterlichen Handschriften. Auch auf anderen Reisen besuchte er Bibliotheken und sah deren Bestände ein, z. B. 1698 in Nürnberg, St. Gallen, Zürich und Bern; vgl. Korzun, Heinrich von Huyssen (wie Anm. 29), S. 28.

31 Als Generaladjutant begleitete er General von Flemming auf dessen Reisen und besuchte 1702 u. a. Karlsbad, Prag, Breslau und Warschau; vgl. Korzun, Heinrich von Huyssen (wie Anm. 29), S. 30.

32 Dies ergibt sich durch die Beschreibung der Handschrift durch den Wittenberger Gymnasiallehrer Wilhelm Ferdinand Wensch (1800-1867), der sie auf der alten Universitätsbibliothek zu Wittenberg eingesehen hatte. Vgl. [W. F.] Wensch, Collation eines Wittenbergischen Codex, in: Zeitschrift für die Alterthumswissenschaft 1838, Nr. 138, Sonntag 18. November, Sp. 1115-1118, Nr. 139, Mittwoch, 21. November, Sp. 1119-1122 (Fortsetzung).

33 Vgl. ein beigelegtes, sich wohl auf diesen Codex beziehendes Übergabeprotokoll, wonach 1 Cod. manuscr., 62 Schr(iften) in 4 u. 14 Schr(iften) in 8 von Karl Heinrich Eduard Lommatzsch (seit 1832 Professor und seit 1838 3. Direktor am Predigerseminar) an Dr. und Oberlebrer Flathe in Planen übergeben werden. Weitere Angaben in der Beschreibung (wie Anm. 26). Zu Flathe vgl. Woldemar Lippert, Heinrich Theodor Flathe und seine Stellung in der sächsischen Geschichtsschreibung, in: Armin Tille (Hg.), Deutsche Geschichtsblätter. Monatsschrift zur Förderung der landesgeschichtlichen Forschung, Bd. 1, Gotha 1900, S. 223-227. 
Der Codex H $2^{34}$ enthält Werke des spätantiken Dichters Boethius, nämlich seine berühmte Consolatio philosophiae (Abb. 4) sowie die Opuscula sacra, und entstand nach Schriftbefund im letzten Viertel des 13. Jahrhunderts, wohl in Südfrankreich oder Norditalien. Die Consolatio ist mit der hauptsächlich in Frankreich verbreiteten Glosse des Wilhelm von Conchis († nach 1154) versehen - dies könnte ebenfalls auf eine Entstehung in (Süd-?)Frankreich deuten. ${ }^{35}$ Wie das auf der letzten Seite eingefügte, wohl einer mitteleuropäischen Schreibhand zuzuweisende Inhaltsverzeichnis zeigt, befand sich der Band schon im späten 14. oder frühen 15. Jahrhundert in Deutschland. Dass die Bindung in einen schlichten Einband $^{36}$ in einem Franziskanerkloster erfolgte, belegt die verwendete Makulatur (die ehemaligen Spiegel), von der heute nur noch Abklatsche (also Abdruckspuren der abgelösten Blätter) vorhanden sind. Es handelt sich um Fragmente des Protokolls eines Ordenskapitels, wahrscheinlich vom Provinzialkapitel der Franziskaner in Frankfurt/Oder im Jahr 1391.37 Außerdem findet sich auf der letzten Seite (S. 132) ein teilweise radierter Besitzeintrag aus dem 3. Viertel des 15. Jahrhunderts: [Beginn, evtl. Vorname, radiert] de Jessen Torgow[e]n[sis]. Der Vorbesitzer stammte demnach aus dem Ort Jessen (Elster) ca. 40 Kilometer nördlich von Torgau und bezeichnete sich als ,Torgauer‘, gehörte also vielleicht dem dortigen Franziskanerkonvent an oder könnte diesem den Band gestiftet haben. Es ist zu vermuten, dass die Handschrift sich damals im Franziskanerkloster Torgau oder einem in der Nähe gelegenen Kloster ${ }^{38}$ befand.

34 Ausführliche Beschreibung der Handschrift unter: http://www.manuscripta-mediae valia.de/dokumente/html/obj31587622.

35 Bei den Marginal-, evtl. auch den Interlinarglossen der ältesten Glossenhand (Glossenhand 1) bestehen Übereinstimmungen zum Kommentar des Guillelmus de Conchis, sodass es sich um Auszüge in Glossenform handeln könnte. Vgl. Éduard Jeaneau (Hg.), Gvillelmi de Conchis Opera omnia, Bd. 2: Lodi Nauta (Hg.), Glosae svper Boetivm, Turnhout 1999 (Corpvs Christianorum. Continuatio medieualis 158), S. 10 15. Zur Überlieferung dieses Kommentars in vollständigen Abschriften, Glossen sowie Auszügen (ohne diese Handschrift) vgl. NautA, ebd., S. LXXX-LXXXII (16 Handschriften, davon mindestens neun mit französischer Provenienz).

36 Der Einband wurde bei einer Restaurierung im Jahr 1969 erneuert, die Reste des historischen Einbandes werden im Schuber aufbewahrt. Demnach war der Holzdeckeleinband mit rotem Leder bezogen, das durch einfache Streicheisenlinien in einen Rahmen und zwei sich in Form eines Andreas-Kreuzes überschneidende Doppellinien gegliedert war; der Band wurde mit einer Langriemenschließe geschlossen.

37 Vgl. die beschnittenen Angaben zum Ausstellungsort: [Act]um et datum in [...] capitul[um] nostr[um] in Frank[e]nfurt anno domini Millesimo iii [...]. In Frankfurt/ Oder befand sich ein Franziskanerkloster, in dem im 14. Jahrhundert nur einmal (1391) ein Provinzialkapitel der Ordensprovinz Saxonia stattfand. Vgl. Tabelle zur Provinzgeschichte in: Volker Honemann (Hg.), Geschichte der Sächsischen FranziskanerProvinz von der Gründung bis zum Anfang des 21. Jahrhunderts, Bd. 1: Von den Anfängen bis zur Reformation, Paderborn 2015, S. 853, 858.

38 In Frage kämen z. B. die Klöster in Oschatz (ca. 35 km südlich), Wittenberg (ca. 50 km nordwestlich), Jüterbog (ca. $60 \mathrm{~km}$ nördlich) oder Meißen (ca. $65 \mathrm{~km}$ südöstlich). 
Am 17. Juni 1691 wurde der Band der Bibliothek der Fürstenschule St. Afra in Meißen durch den einstigen Schüler Christian Friedrich Ilmann gestiftet. ${ }^{39}$ Ilmann war 1652 in Tanneberg bei Meißen geboren worden und von 1667 bis 1672 Schüler an der Fürstenschule St. Afra. Er studierte seit 1672 an der Universität Leipzig und war seit 1677 Pfarrer von Neukirchen/Erzgebirge; er starb 1710.40 Wie Ilmann in den Besitz dieses Bandes gekommen ist, ließ sich nicht ermitteln. Eine erste Beschreibung der Handschrift fertigte 1770 der Rektor von St. Afra Johann Gottfried Hoere (1755-1771) an. ${ }^{41}$

Ebenfalls durch eine Stiftung erhielt die Bibliothek von St. Afra eine großformatige, prachtvoll illuminierte Bibel (H 4).42 In der Literatur wurde angenommen, dass es sich dabei um ein Überbleibsel aus dem Meißner Chorherrenstift St. Afra handele. ${ }^{43}$ Tatsächlich aber wurde dieser Band der Schule um die Mitte des 17. Jahrhunderts vom kurfürstlichen Bibliothekar in Dresden Christian Brehme $(† 1667)$ geschenkt. ${ }^{44}$ Dies geht aus der 1756 vom Rektor Johann Gottfried Hoere

39 Vgl. die Widmungsinschrift auf dem vorderen Spiegel: Afrae / Misnensium Inclite / Studiorum juventute / Matri / optime et dexterrime / ejusque Bibliothece / consecrat Hoc MSS. et offert / in grati animi monumentum / illius olim filius / et per sexennium ferme Alumnus / nunc / Neukirchensium Dioecesenis Misnensis / Pastor: / ChristianFridericus Ilmann / xv Calend: Julij / Anno MDCXCI [17. Juni 1691].

40 Zur Biografie vgl. u. a. Reinhold Grünberg, Sächsisches Pfarrerbuch. Die Parochien und Pfarrer der Ev.-luth. Landeskirche Sachsens (1539-1939), 2 Bde., Freiberg 1940, Bd. 1, S. 387, sowie Pfarrerbuch Sachsen: http://pfarrerbuch.de/sachsen/pfarrer/ christian-friedrich-ilmann-1652-1710/.

41 Johann August Müller, Versuch einer vollständigern Geschichte der Chursächsischen Fürsten- und Landschule zu Meissen [...], Leipzig 1789, Bd. 1, S. 141, verweist auf: Johann GotTfried Hoere, De donatis bibliothecae Afranae Boethii scriptis, Misenae 1770. Das Werk konnte bislang weder in den Katalogen der Bibliotheksverbünde (KVK, VD18) noch in der HeBis-Datenbank der Schulprogramme ermittelt werden.

42 Ausführliche Beschreibung unter: http://www.manuscripta-mediaevalia.de/doku mente/html/obj31589610.

43 So z. B. Johann Friedrich Ursinus, Historische Untersuchung des Ursprungs der Kirche und des Klosters Sanct Afra in der Stadt Meißen [...], Leipzig 1780, S. 84: die in der dasigen Fürstenschulbibliothek befindliche und aus der alten Klosterbibliothek gerettete und sauber geschriebene Bibel in Folio. Eine Herkunft aus dem Chorherrenstift St. Afra wird z. T. auch noch in der jüngeren Literatur angenommen; so bei BRÜCK, Geschichte (wie Anm. 23), S. 19, 63, sowie Dirk Martin Mütze, Das AugustinerChorherrenstift St. Afra in Meißen (1205-1539) (Schriften zur sächsischen Geschichte und Volkskunde 54), Leipzig 2016, S. 210: „Möglicherweise stammt eine im Meißner Stadtarchiv aufbewahrte Bibelhandschrift aus der Stiftsbibliothek", hier auch zu zumeist liturgischen Fragmenten, die evtl. aus der Stiftsbibliothek stammen und als Akteneinbände verwendet wurden.

$44 \mathrm{Zu}$ Christian Brehme (geboren 1613 in Leipzig, Studium der Philosophie, Politik und Jura in Wittenberg und Leipzig, 1640 bis 1654 kurfürstlicher Bibliothekar in Dresden, seit 1642 Mitglied des Dresdner Rats und seit 1657 viermal amtierender Bürgermeister, seit 1660 kurfürstlicher Rat, $† 1667$ in Dresden) vgl. Reinhardt Eigenwill, Christian Brehme, in: Sächsische Biografie, hrsg. vom Institut für Sächsische Geschichte und Volkskunde, Online-Ausgabe: http://www.isgv.de/saebi/, mit weiterer Literatur. 
veröffentlichten Beschreibung der Bibel hervor. ${ }^{45}$ Die um 1469 wohl in Böhmen (s. u.) von einem Schreiber ${ }^{46}$ angelegte großformatige Handschrift (Format 41,5 x $29 \mathrm{~cm}, 389$ Blätter) enthält das Alte und Neue Testament (Bl. 1 19-354v), ein Register zur Einteilung der Epistel- und Evangelienlesungen im Jahresverlauf (Bl. $\left.356^{\mathrm{ra}}-361^{\mathrm{rb}}\right)$ sowie einen häufig in Bibeln überlieferten Kurztext zur Erklärung hebräischer Namen (Bl. 361va-388vb).47 Die Handschrift wurde besonders prächtig mit Buchschmuck ausgestattet. Am Beginn des Alten Testaments (Bl. 1r, Abb. 5) findet sich eine Zierseite, die ein Autorenbild des Hl. Hieronymus (als Verfasser des Prologs) zeigt; das Buch Genesis beginnt mit einer Zierseite (Bl. 3v), welche die Schöpfungsgeschichte in sieben goldgerahmten Medaillons darstellt. ${ }^{48}$ Die einzelnen Bibelbücher werden durch ornamentale und historisierte Initialen ${ }^{49}$ eingeleitet, die Szenen der biblischen Geschichte illustrieren. Beispielhaft zeigt dies etwa der Beginn des Buchs Esther (Blatt 155r), dessen Prolog mit einer Ornamentinitiale und dessen Textbeginn mit einer historisierten Initiale, darstellend das Gastmahl des Königs Ahasver, verziert sind (Abb. 6). Im Neuen Testament finden sich Evangelistenbilder sowie Darstellungen der Apostel. Der zeitgenössische Einband verweist ebenfalls auf den Inhalt, da er mit aus Elfenbein geschnitzten Eckbeschlägen versehen ist, welche die Evangelistensymbole darstellen.

45 Erwähnung der Schenkung durch Christian Brehme an den Rektor der Fürstenschule Andreas Lindenmuth (1642-1664) sowie Beschreibung der Handschrift bei JoHANN GotTfried Hoere, Donata bibliothecae Afranae Biblia Latina describit, Misenae 1756 (Digitalisat: http://digital.slub-dresden.de/werkansicht/dlf/82039/1/), S. 1.

46 Am Ende des Alten Testaments (287vb) findet sich ein (nachgetragener?) Vermerk, der wohl als Schreiber- oder Rubrikatoreintrag zu deuten ist: 1469 per manus Thabor. Er ist vielleicht auf die ca. $85 \mathrm{~km}$ südlich von Prag gelegenen Kleinstadt Tábor zu beziehen. Hoere, Biblia (wie Anm. 45), S. 1, gab an, dass das Alte Testament 1469 von einem Thabor und das Neue Testament im Jahr danach von einem Martin geschrieben worden sei (Libros Veteris Instrumenti [...] ductu literarum, quo monachi solebant uti, exarauit manus Thaboris anno [M] CCCCLXIX, Foederis Noui similiter post calamus Martini). Der Schreibereintrag eines Martinus ließ sich im Neuen Testament jedoch nicht auffinden, auch scheint die Handschrift durchgängig von einer Hand angelegt worden zu sein.

47 Interpretationes nominum hebraicorum sacrae scripturae (Interpretationes, Aaz apprebendens'). Der Text ist in zahlreichen Bibeldrucken enthalten und wird z. T. Stephan Langton zugeschrieben. Weitere Literaturverweise in der Beschreibung (wie Anm. 42).

48 Eine wohl ähnlich prachtvoll gestaltete Zierseite zum Neuen Testament (vor Bl. 288) ging hingegen bereits vor 1756, also vor der Beschreibung durch Rektor Hoere (vgl. Anm. 45), verloren.

49 Im Alten Testament $\left(1^{\mathrm{ra}}-287^{\mathrm{vb}}\right) 65$ ornamentale und 15 historisierte Initialen, im Neuen Testament (287 $\left.7^{\mathrm{vb}}-354^{\mathrm{vb}}\right) 30$ (ursprünglich 32 ) ornamentale und elf (ursprünglich 12 historisierte) Initialen; vgl. die Einzelnachweise mit Darstellung der Ikonografie in der Beschreibung (wie Anm. 42). Durch vor 1756 erfolgte Blattverluste gingen vor Bl. 288 die Zierseite am Beginn des Neuen Testaments mit dem Evangelistensymbol des Matthäus sowie vor Bl. 331 und 347 zwei Zierseiten mit Initialen zu den Philipper- und Kolosser-Briefen und zum Jakobus-Brief verloren. 
Die qualitätvolle Buchmalerei wurde von dem hauptsächlich in Hradec Králové (Königgrätz) tätigen Buchmaler Jan Mikuš ausgeführt. ${ }^{50}$ Er dürfte um 1435 geboren sein, ist 1461 an der Prager Universität nachweisbar und starb nach 1484. ${ }^{51}$ Bislang waren der Forschung vier von Jan Mikuš geschriebene und ausgestattete Graduale- und Antiphonar-Handschriften bekannt. ${ }^{22}$ Die bisher noch nicht im Zusammenhang mit seinem CEuvre gesehene Meißner Bibel umfasst ikonografisches Material zu biblischen Szenen, die in den restlichen Codices aus seinem Atelier nicht enthalten sind. Zu einer Entstehung und Ausstattung in Böhmen passen auch die wenigen alttschechischen Marginalglossen, ${ }^{53}$ die zeigen, dass die Bibel bald nach ihrer Entstehung in Böhmen studiert wurde. Bislang ist nicht bekannt, wie Christian Brehme in der Mitte des 17. Jahrhunderts in den Besitz dieser wertvollen Bibel aus dem böhmischen Raum gelangte.

Eine volkssprachige Handschrift medizinischen Inhalts, die ebenfalls aus der Schulbibliothek von St. Afra stammt, soll unten in Kapitel IV vorgestellt werden.

\section{Handschriftenfragmente}

Neben den vollständigen Codices werden im Meißner Stadtarchiv auch Handschriftenfragmente aufbewahrt. Es handelt sich um 42 Pergamentblätter, die vor der Bearbeitung provisorisch sortiert und in zwei Mappen aufbewahrt waren. Im Zuge der Erschließung wurden zusammengehörige Stücke unter einer Signatur vereint (Fragment 1-28), ${ }^{54}$ außerdem wurde 2017 in der Restaurierungsabteilung der Universitätsbibliothek Leipzig eine optimalere Lagerung vorgenommen. Die

50 Den Hinweis auf diesen Buchmaler verdanke ich Dr. Armand Tif (Universität Wien), dem ich zu großem Dank verpflichtet bin.

51 Zum Maler vgl. Barry F. H. Graham, Bohemian Scribes and Illuminators in the Jagellionian Era (1471-1525), in: Scriptorium 54 (2000), S. 319-341, hier S. 320-322 sowie Kat. S. 333 f. Zu weiterer Literatur vgl. die Beschreibung (wie Anm. 42).

52 Hradec Králové, Ostböhmisches Museum (Muzeum východních Čech), Hr 2 (olim II A 2): Graduale, geschaffen für die utraquistische Hl.-Geist-Kirche in Hradec Králové, mit Kolophon des Jan Mikuš (317v, abgedruckt bei Graham, Bohemian Scribes (wie Anm. 51), S. 321; Barry F. H. Graham, Bohemian and Moravian Graduals 1420-1620, Turnhout 2006, Nr. 14, S. 170-172), Hr 3 (olim II A 3): Antiphonar, Hr 4 (olim II A 4), Antiphonar, Prag, NB, XIV.A.1: Graduale civitatis Gurimensis, geschaffen 1470 wohl für die utraquistische Kirche St. Bartholomäus in Kolin, erst seit 1573 in Kouřim, mit hexametrischem Kolophon des Malers Johannes Mikuš (1 1 r: Hunc Johannes Mikus fecit de Hradecz ortus, abgedruckt bei Graham, Bohemian Scribes (wie Anm. 51), S. 321 und Graham, Graduals (a. a. O.), S. 360). Weiterer Literatur und Verweise auf OnlineBeschreibungen und Digitalisate in der Beschreibung (wie Anm. 42).

53 Transkription und Übersetzung der alttschechischen Glossen (Bl. 17va $159^{\mathrm{vb}}, 162^{\mathrm{rb}}$, $164^{\mathrm{rb}}, 219^{\mathrm{rb}}$ ) in der Beschreibung (wie Anm. 42). Dr. Thomas Krzenck (Leipzig) ist für die Hinweise zu den alttschechischen Glossen sehr herzlich zu danken (schriftliche Mitteilungen vom 5. und 9. März 2017).

54 Die ausführlichen Fragmentbeschreibungen können über die Projektseite (wie Anm. 2) eingesehen werden. 
Ablösung der meisten Fragmente von den Akten des Meißner Stadtarchivs erfolgte sehr wahrscheinlich 1945 oder 1946 auf Veranlassung des damaligen Stadtarchivars Dr. Helmuth Gröger (1890-1957). ${ }^{55}$ Zwei Fragmente stammen aus Drucken: Fragment 27 überliefert zwei Doppelblätter und ein Einzelblatt des 1485 gedruckten Missale Misnense, ${ }^{56}$ Fragment 28 zwei Einzelblätter eines bislang unidentifizierten Frühdrucks des Missale Franciscanum, der im frühen 16. Jahrhundert wohl in Frankreich oder den südlichen Niederlanden entstand und eine qualitätvolle Ausstattung erfuhr. Die restlichen Blätter (Fragment 1-26) stammen aus Handschriften, wobei nur Fragment 26 deutsche Texte ${ }^{57}$ und die restlichen lateinische Texte überliefern. Neben theologischen (Fragment 1-5) handelt es sich um liturgische Fragmente.

Wie aus den Aktenaufschriften hervorgeht, wurden 17 der 28 Fragmente in der Zeit zwischen 1556 und 159958 als Umschläge von Jahrrechnungen bzw. Stadtoder Zins-Registern der Meißner Stadtverwaltung59 verwendet, zwei Fragmente tragen jüngere Aktenaufschriften (von 1621/22 und 1659). ${ }^{60}$ Der Meißner Stadtrat und die zuständigen Schreiber und Archivare verwendeten in der Zeit zwischen der zweiten Hälfte des 16. und dem zweiten Drittel des 17. Jahrhunderts also regelmäßig Pergamentblätter als Aktenumschläge. Es ist anzunehmen, dass man die dafür benötigten Pergamentblätter nicht von außerhalb einführte, sondern auf Material zurückgriff, das vor Ort vorhanden war. Möglicherweise stammen also zumindest einige dieser zweitverwendeten, „recycelten“ Pergamentblätter aus Handschriften derjenigen geistlichen Institutionen in Meißen und der Umgebung, die in der Reformationszeit aufgelöst worden waren, also z. B. aus dem 1539 aufgelösten Franziskanerkloster in Meißen oder dem 1540 bis 1544 säkularisierten Zisterzienserkloster in Altzelle.

55 Freundliche Auskunft von Tom Lauerwald (Stadtarchiv Meißen). Zur Biografie Grögers (1929 bis 1946 Stadtarchivar und 1948 bis 1951 Mitarbeiter im Stadtarchiv) vgl. StefFen Förster, Helmuth Gröger, in: Sächsische Biografie (wie Anm. 44).

56 Missale Misnense. Hrsg. von Johann (von Weissenbach), Bischof von Meissen. Mainz: Peter Schöffer, 27.VI.1485. Vgl. Gesamtkatalog der Wiegendrucke (im Folgenden: GW) M24543: http://gesamtkatalogderwiegendrucke.de/docs/M24543.htm. Es handelt sich um einen Pergamentdruck mit rot gedruckten Rubriken.

57 Das kleinformatige Blatt aus dem 1. Drittel des 15. Jahrhunderts enthält deutsche katechetische Texte (Pater noster, Ave Maria und Credo) und ist aufgrund der Schreibsprache in Thüringen oder Sachsen entstanden. Das Blatt wurde wahrscheinlich im 19. Jahrhundert aus einer Handschrift ausgeschnitten, die in den Besitz eines englischen Sammlers gelangte. Vgl. die Beschreibung von Werner Hoffmann: http:// www.manuscripta-mediaevalia.de/dokumente/html/obj31593012.

58 Die ältesten, auf 1556 datierten Aktenaufschriften finden sich auf Fragment 11 und 15, eine auf 1599 datierte Aufschrift auf Fragment 5.

59 Z. B. Fragment 6: Stadt Rechnung zw Meissen Undt beschlossen 1595, Fragment 12: Jahr Rechnung der Stadt Meissen 1593, angefangen Walpurgis 93. Undt beschlossen 94, Fragment 27: Zinß Register des Raths zu Meissen uf das 1571. 72. 73. 74. 75. und 76. Jabr.

60 Fragment 34 und 23. 
Untersucht man daraufhin den Inhalt der liturgischen Fragmente, so folgen mindestens fünf Bruchstücke aus verschiedenen Missale-Handschriften des 14. und 15. Jahrhunderts (Fragment 10-13 und 15) nach Textvergleich dem franziskanischen Ordo ${ }^{61}$ und könnten also aus dem Meißner Franziskanerkloster stammen. Zwei Lektionar-Fragmente des 13. Jahrhunderts (Fragment 16 und 17) weisen in der Abfolge der Lektionen Übereinstimmungen mit dem Zisterzienser-Brevier auf. ${ }^{2}$ Sie könnten also aus Altzelle oder einem anderen sächsischen Zisterzienserkloster stammen. Das etwas ältere, in das 1. Viertel des 13. Jahrhunderts zu datierende Fragment 16 (Abb. 7) enthält die Vorrede des Hieronymus sowie den Beginn des Buchs Genesis, eingeteilt in zwölf Lesungen für die Sonntage vor der Fastenzeit (Septuagesima und Quinquagesima). Jede Lesung wird durch eine für den zisterziensischen Initialstil typische grün-rote Silhouetteninitiale eingeleitet. Die Textabschnitte sind für die Lesung durch verschiedene Zeichen (die sogenannte „Zisterzienserinterpunktion“) 63 gegliedert.

Wohl aus einem Kloster der Zisterzienser oder Benediktiner stammt ein in die zweite Hälfte des 14. Jahrhunderts zu datierendes Doppelblatt (Fragment 24), das Auszüge aus dem Ordo zur Weihe und Einkleidung von Mönchen und Nonnen enthält. Die Ordnung der Jungfrauenweihe wird sehr ausführlich geregelt und umfasst die zeremoniellen Akte der Übergabe des Schleiers, der Krone und des Rings. Deshalb ist davon auszugehen, dass das Blatt entweder aus einem für ein Frauenkloster angelegten Rituale oder aus einem für den Bischof geschriebenen Pontifikale stammt.

Bemerkenswert ist ein 1590 als Umschlag einer Meißner Stadtrechnung verwendetes Doppelblatt eines Liber ordinarius aus der zweiten Hälfte des 15. Jahrhunderts (Fragment 25), der wahrscheinlich für den Meißner Dom angefertigt wurde (Abb. 8). Dass das Blatt aus einem Ordinarius stammt, der den Gottesdienst an einer Stiftskirche regelte, zeigt die Erwähnung von Prozessionen zu verschiedenen Kapellen und Altären, von Vikaren und einem provisor, von liturgischer Bekleidung (so dem Chormantel, der cappa) und dem Chorgestühl.64 Auf den Meißner Dom verweisen die Erwähnung der Fürstenkapelle (capella dominorum

61 Die Übereinstimmungen betreffen einerseits die Abfolge der Gesänge und Lesungen, andererseits wörtliche Übereinstimmungen bei den liturgischen Rubriken. Zum Textvergleich wurde herangezogen: STEPHEN J. P. van DijK (Hg.), Sources of the modern Roman liturgy. The ordinals of Haymo of Faversham and related documents (1243-1307) (Studia et documenta Franciscana 1-2), 2 Bde., Leiden 1963.

62 Die Abfolge der Lektionen stimmt mit der „bernhardinischen“ Rezension des Zisterzienserbreviers (nach 1175) überein. Überprüft am Digitalisat der Handschrift Dijon, Bibliothèque municipal, Ms. 114 (geschrieben 1183 bis 1188 in Cîteaux), 58 $8^{\mathrm{r}-\mathrm{v}}$ : http:// patrimoine.bm-dijon.fr/pleade/ead.html?c=FR212316101_citeaux_D11010274.

63 Vgl. dazu Nigel F. Palmer, „Simul cantemus, simul pausemus“. Zur mittelalterlichen Zisterzienserinterpunktion, in: Eckart Conrad Lutz u. a. (Hg.), Lesevorgänge. Prozesse des Erkennens in mittelalterlichen Texten, Bildern und Handschriften (Medienwandel - Medienwechsel - Medienwissen 11), Zürich 2009, S. 483-570, hier S. 485 f.

Einzelnachweise in der Beschreibung. 
ducum $)^{65}$ und anderer liturgischer Orte ${ }^{66}$ sowie für das Bistum typischer Festtage. ${ }^{67}$ Diese Verweise gestatten auch eine Eingrenzung der Datierung auf zwischen 1440 und 1485.68 Offensichtlich handelt es sich um eine individuelle Zusammenstellung eines Kantors oder Succentors für das Chorgebet und die Messfeier im Meißner Dom. ${ }^{9}$ Dass dieser Ordinarius nur fragmentarisch erhalten ist, ist angesichts seines Aussagewertes zur spätmittelalterlichen Meißner Domliturgie als großer Verlust anzusehen.

Angesichts der fast vollständigen Verluste der liturgischen Überlieferung aus den sächsischen Klöstern und Stiftskirchen durch die Ereignisse der Reformationszeit ${ }^{70}$ sind die Meißner Fragmente als potenzielle Reste der liturgischen Schriftlichkeit dieser geistlichen Einrichtungen von Bedeutung. Andere Fragmente sind hingegen inhaltlich zu wenig spezifisch, als dass man sie einem konkreten Bistum oder Kloster zuordnen könnte.

Das umfangreichste Fragment der Sammlung (Fragment 3) besteht aus sechs Doppel- und zwei Einzelblättern und überliefert Auszüge aus der Postilla zur Bibel des Dominikaners Hugo von Saint-Cher (de Sancto Caro, $\dagger 1263$ ). Nach

65 Die um 1420 unter Markgraf Friedrich dem Streitbaren ( $†$ 1428) begonnene und 1440 unter seinem Sohn Friedrich dem Sanftmütigen $(\dagger 1464)$ vollendete, an die Westfront des Meißner Doms angebaute Fürstenkapelle diente als Begräbniskapelle der Wettiner. In ihr vollzogen zunächst zwei Vikare den Altardienst; ihre Zahl wurde 1445 auf sieben Vikare erhöht, denen sieben Choralisten zur Hilfe beigegeben wurden. Vgl. KunZ voN Brunn, gen. von Kauffungen, Das Domkapitel von Meißen im Mittelalter. Ein Beitrag zur Verfassungs- und Verwaltungsgeschichte der deutschen Domkapitel, in: Mitteilungen des Vereins für Geschichte der Stadt Meißen 6 (1904), S. 121-253, hier S. 166 f.; Hermann Klemm, Zur Geschichte des Gottesdienstes im Dom zu Meißen während des Mittelalters, in: Franz Lau (Hg.), Das Hochstift Meißen. Aufsätze zur sächsischen Kirchengeschichte, Berlin 1973 (Herbergen der Christenheit. Sonderband), S. 123-145, hier S. $132 \mathrm{f}$.

66 Die Prozession (Bl. 2 ${ }^{\text {vb }}$ ) führte u. a. zu der als Kapitelsaal dienenden Allerheiligenkapelle im Kreuzgang, zu der an der Westfront des Doms angebauten Fürstenkapelle (in capella dominorum ducum) sowie zu dem Kreuzaltar in der Mitte der Kirche.

67 Am oberen Blattrand von $1^{\mathrm{v}}$ fügte ein jüngerer Schreiber einen Nachtrag zur Oktav der Hll. Fabian und Sebastian (27. Januar) ein, die nach Grotefend nur im Bistum Meißen gefeiert wurde.

68 Die erwähnte Fürstenkapelle wurde 1440 vollendet, das in der vorherigen Anmerkung genannte, in der Handschrift aber nachgetragene Fest nach 1485, aber vor 1502 eingeführt; vgl. die Ausführungen in der Beschreibung.

69 Der Ordinarius war Teil einer umfangreicheren liturgischen Handschrift, wie sich aus Seitenverweisen (z. B. $2^{\text {ra: }}$ in fine libri bzw. require xxui) ergibt. Die Gesänge und Gebete wurden jeweils nur anzitiert, dafür finden sich teilweise ausführliche liturgische Rubriken, in denen auf örtliche Gegebenheiten (Kapellen und Altäre) Bezug genommen wird. Hier finden sich auch Verweise auf die am jeweiligen Festtag zu lesenden Predigten $\left(2^{\mathrm{ra}}\right)$ sowie auf eine Legenda zu Corpus Christi am Ende einer PassionaleHandschrift $\left(2^{\mathrm{rb}}\right)$.

$70 \mathrm{Zu}$ den Verlusten der liturgischen Überlieferung aus sächsischen Klöstern vgl. Christoph Mackert, Geist aus den Klöstern. Buchkultur und intellektuelles Leben in Sachsen bis zur Reformation, Katalog zur gleichnamigen Ausstellung der Universitätsbibliothek Leipzig vom 13. Oktober 2017 bis 7. Januar 2018, Leipzig 2017, hier S. 54 f. 
Ausweis des Schriftbefunds entstand die Ursprungshandschrift in der 2. Hälfte des 13. Jahrhunderts, wohl in Frankreich. Weiterhin ist unter den theologischen Fragmenten ein Doppelblatt mit einem Ausschnitt aus der Summa (Quaestiones ordinariae) des in Paris, Brügge und Tournai wirkenden scholastischen Autors Heinrich von Gent $(† 1293)$ hervorzuheben (Fragment 4, vgl. Abb. 9). Das Blatt dürfte nach Ausweis der charakteristischen, stark gekürzten Kursive („Anglicana book hand“) im 1. Viertel des 14. Jahrhunderts in England oder Nordwestfrankreich entstanden sein. Es handelt sich damit um einen neuen und autornahen Textzeugen des nicht allzu häufig überlieferten Werks. ${ }^{71}$ Anders als die restlichen Stücke diente dieses Fragment nicht als Aktenumschlag, sondern war auf zwei Pappdeckel gezogen ${ }^{72}$ und diente wohl seit dem 17. Jahrhundert als Bezug eines Buchs, vielleicht ebenfalls aus der Bibliothek der Fürstenschule St. Afra. ${ }^{73}$

\section{Lateinisch-deutsche Rezeptsammlung - das Manual eines (Meißner?) Wundarztes}

Der Inhalt der Handschrift H 6, die wie H 1-4 (s. o.) aus der Bibliothek der Fürstenschule St. Afra stammt und 1948 in das Stadtarchiv gelangte, wird von Beate Brück in ihrer Diplomarbeit zur Geschichte der Bibliothek von St. Afra sehr summarisch umschrieben mit „Arzneibuch. Papierhandschrift des 15. oder 16. Jahrhunderts“ ${ }^{74}$ Eine nähere Untersuchung ${ }^{75}$ ergibt zunächst, dass der Band aus drei ursprünglich selbstständigen Faszikeln besteht, zwei handschriftlichen Teilen (I-II) und einem Druck (III), die alle ungefähr zur gleichen Zeit, um 1485, entstanden sind: Faszikel I (Blatt 1-192) enthält eine lateinisch-deutsche Rezeptsammlung, Faszikel II (Bl. 193-200) ein lateinisches Zodiakalmondbuch, ${ }^{76}$ Faszikel III

71 Zur Textüberlieferung vgl. die Edition: Raymund Macken (Hg.), Henrici de Gandavo Opera omnia, Bd. 27: Summa (quaestiones ordinariae), art. XXXI-XXXIV, Leuven 1991, hier S. XXXVII-XLIV (18 Handschriften, die articulus 31-34 der Summa überliefern).

72 Nach Rücksprache mit dem Stadtarchiv Meißen wurde das Fragment im April 2017 in der Restaurierungswerkstatt der Universitätsbibliothek Leipzig von den Pappdeckeln abgelöst.

73 Vgl. die kopfständige Signatur am oberen Rand von 1v: Lit: T. [gestrichen:] No: 24, darunter: Nō 7. Auf dem ehemaligen Rücken alte Bandnummer: 26 oder 20. Die Signatur lässt vermuten, dass der Trägerband aus der Bibliothek der Fürsten- und Landesschule St. Afra in Meißen stammte, wo unter der Signatur T die Saxonica aufgestellt waren; vgl. BRÜCK, Geschichte (wie Anm. 23), S. 39.

74 BRÜck, Geschichte (wie Anm. 23), S. 20.

75 Ausführliche wissenschaftliche Beschreibung unter: http://www.manuscripta-mediae valia.de/dokumente/html/obj31591356 (dort auch weiterführende Literatur zu den erwähnten Personen).

76 Zur Parallelüberlieferung vgl. Lynn Thorndike/Pearl Kibre, A Catalogue of Incipits of Mediaeval Scientific Writings in Latin. Revised and Augmented Edition, London 1963, Sp. 834,7; zur Textgattung B. BRÉVART, Mondwahrsagetexte (deutsche), in: Verfasserlexikon (wie Anm. 3), Bd. 6 (1987), Sp. 674-681. 
(Bl. 201-238) einen 1484 bei Markus Brandis in Leipzig erschienenen medizinischen Druck: Albicus von Unicow: Regimen hominis sive Vetularius (GW 804). ${ }^{77}$ Im Folgenden soll nur auf Faszikel I eingegangen werden, da dieser in dem Sammelband den weitaus größten Raum (192 von 238 Blättern) einnimmt und die beiden anderen Teile nur als eine Art Anhang fungieren.

Teil I enthält eine umfangreiche, inhaltlich überaus reichhaltige, in ihrem Kernbestand $\left(1^{\mathrm{r}}-157^{\mathrm{v}}\right)$ aus ca. 570 Stücken bestehende Rezeptsammlung. Laut Wasserzeichenbefund ist der Teil um 1485 entstanden, aufgrund der Schreibsprache im obersächsischen Sprachraum. Als Entstehungsort kommt in erster Linie Meißen in Frage, daneben vielleicht auch Leipzig.

Für Meißen als mögliche Heimat des Schreibers und Kompilators der Rezepte, der seinen Namen an keiner Stelle nennt, spricht die Art, wie er den Meißner Domherrn Hildebrand zitiert: Bei dem Rezept auf Bl. $8^{\mathrm{v}}$ verweist er nur auf einen dominus doctor Hildebrand als Autor, so als ob er voraussetzte, dass dieser allgemein bekannt sei. Auf Bl. $145^{\mathrm{v}}$ stellt er ihn dann ausführlich als Kanoniker des Meißner Doms und Leibarzt der Herzöge von Sachsen vor: Sequentia [d. h. die folgenden 21 Rezepte] habeo a domino doctore Hildebrando viro perito, phisico ducum Saxonie et ecclesie Mißnensis canonico. Der Rezeptautor ist zu identifizieren mit Hildebrand Guntheri de Northeym; dieser immatrikulierte sich im Wintersemester 1436/37 an der Universität Erfurt und wurde dort 1440 Baccalaureus, 1444 wurde er an der Universität Pavia zum Doktor der Medizin promoviert, 1451 war er Rektor an der Universität Erfurt, 1462 Kanoniker am Dom zu Meißen und an der Marienkirche in Erfurt, am 9. März 1483 starb er als Domherr zu Meißen.78 Dass er Leibarzt der Herzöge von Sachsen war, wird anscheinend außer in dieser Handschrift nirgendwo erwähnt. Der enge Bezug des Schreibers zu Meißen zeigt sich insbesondere darin, dass er sich bei den biografischen Daten des Hildebrand auf Meißen beschränkt und nicht erwähnt, dass dieser auch Kanoniker an der Marienkirche in Erfurt war und sogar Rektor der Universität Erfurt.

Ein weiterer Rezeptautor aus Meißen wird auf Bl. $47^{\mathrm{r}}$ angeführt: $A$ domino magistro Jacobo Zadel de Mißnia habeo sequentes sentencias. Dabei handelt es sich um Iacobus Czadil de Misna, der im Wintersemester 1444 an der Universität Leipzig immatrikuliert, im Wintersemester 1446 dort zum Baccalaureus und im Wintersemester 1462 zum Magister promoviert wurde..$^{79}$ Von einer Tätigkeit des Jacobus als Mediziner war bislang nichts bekannt.

Aus einer Bemerkung gleich am Anfang (1 $\left.1^{\mathrm{r}}\right)$ geht hervor, dass der anonyme Kompilator/Schreiber der Sammlung als Wundarzt in Leipzig praktizierte; er

77 Zu dem Druck vgl. GW, Bd. 1, Leipzig 1925, Nr. 804; mit Nachweis von 12 Exemplaren; http://gesamtkatalogderwiegendrucke.de/docs/GW00804.htm, mit Nachweis von 22 Exemplaren, darunter auch das des Meißner Stadtarchivs.

78 Zur Biografie Hildebrands vgl. ERICH Kleineidam, Universitas studii Erffordensis, Bd. 1 (Erfurter theologische Studien 14), Leipzig 21983, S. 358.

79 Zur Biografie Jacob Zadels vgl. ErLer, Matrikel (wie Anm. 7), Bd. 1, S. 150, Bd. 2, S. 139,188 , Bd. 3, S. 971. 
rühmt sich, dort eine Fistel geheilt zu haben, an der sich bereits viele vergeblich versucht hätten: et specialiter fistulam in Liepczk curaui, in qua plures manum apposuerunt, proficere autem illi non potuerunt. Dies muss jedoch nicht heißen, dass er in Leipzig wohnhaft war, die Formulierung in Liepczk curaui deutet eher darauf hin, dass er sich zur Zeit der Niederschrift an einem anderen Ort als Leipzig aufhielt und auf die medizinischen Erfolge, die er dort hatte, verweist.

In der Sammlung werden auffällig viele Rezeptautoren genannt. Darunter befinden sich nur ganz wenige bekannte Verfasser medizinischer Werke. ${ }^{80}$ Es handelt sich vielmehr fast durchgängig um Gewährsmänner, von denen der Schreiber die Rezepte persönlich erhielt: Immer wieder gebraucht er die Ausdrücke babeo a, babui a oder acquisivi a. ${ }^{81}$ Dass die Vermittlung der Rezepte mehrheitlich nicht auf brieflichem Wege geschah, sondern durch persönlichen Kontakt, darauf deuten die genaueren geografischen Angaben, die auf einen Aufenthalt an den jeweiligen Orten schließen lassen, wie Scheuwern [Scheyern] v meil von Munchen (23r), Friburga 6 miliaribus a Basilea (27r); in Tannis [Thann, Oberelsass] v miliaribus de Basilea (46v), in monasterio Seitz in Sclanonia [Kartäuserkloster Seitz in Slowenien] ii miliaribus a Cili [Celje] prope regnum Bosnie (100r). Auf eine Reisetätigkeit des Schreibers deuten auch einige Stellen, an denen er Gastwirte als seine Gewährsmänner bezeichnet. ${ }^{82}$

Wie sich aus den genannten Wohnorten der Rezeptautoren und Gewährsmänner ergibt, muss er bei seiner Tätigkeit als Wundarzt große Teile Süd- und Mitteldeutschlands (einschließlich Österreichs) bereist haben. Er nennt folgende 25 Orte (in alphabetischer Reihenfolge): Bamberg (151 $\left.{ }^{\mathrm{r}}, 156^{\mathrm{r}}\right)$, Basel $\left(46^{\mathrm{v}}\right)$, Bautzen $\left(48^{\mathrm{r}}\right.$, 63v), Bebenhausen (46v), Brixen (144v), Coburg (10r), Debingen [Tübingen?] (46v), Eichstätt (45v), Erfurt (17v , 45v , 48 $\left.{ }^{\mathrm{r}}, 112^{\mathrm{v}}\right)$, Frankfurt a. M. (53v, $\left.54 \mathrm{r}\right)$, Freiburg i. Br. $\left(27^{\mathrm{r}}\right)$, Heisterbach $\left(51^{\mathrm{v}}\right)$, Leipzig $\left(1^{\mathrm{r}}, 85^{\mathrm{v}}\right)$, Lichtenberg $\left(27^{\mathrm{v}}\right)$, Luckau $\left(3^{\mathrm{r}}\right)$, Mainz (49v $\left., 51^{\mathrm{v}}, 76^{\mathrm{v}}\right)$, Meißen $\left(47^{\mathrm{r}}, 145^{\mathrm{v}}\right)$, Regensburg (135r), Reichenau an der Rax [südl. von Wien] (19v), Scheyern (23r), Seitz [Žiže] in Slowenien (100r), Straßburg (1 ${ }^{\mathrm{r}}, 9^{\mathrm{v}}$, 10r $\left., 15^{\mathrm{v}}, 27^{\mathrm{r}}, 28^{\mathrm{v}}, 48^{\mathrm{r}}, 76^{\mathrm{v}}\right)$, Wertheim $\left(46^{\mathrm{v}}\right)$, Wien $\left(88^{\mathrm{v}}\right)$, Würzburg $\left(45^{\mathrm{v}}, 145^{\mathrm{v}}\right)$.

80 An bekannten medizinischen Autoren werden genannt: Johannes de Gadesden $\left(4^{\mathrm{r}}\right)$, Arnaldus de Villanova $\left(4^{\mathrm{v}}\right)$, Johannes de Janua $\left(56^{\mathrm{r}}\right)$, Johannes de Sancta Sophia (57v). Insgesamt enthält die Handschrift nur wenige näher identifizierbare Texte, darunter $88^{\mathrm{v}}-99^{\mathrm{r}}$ als einzigen längeren Text ein alphabetisches Krankheitenverzeichnis, überschrieben mit Egritudines tocius corporis cum descripcionibus et remediis eorundem secundum ordinem alphabeti (zum Text vgl. GW (wie Anm. 77), Nr. 8255; Thorndike/ KibRe, Incipits (wie Anm. 76), Sp. 85,7).

81 Beispiele: Habeo a magistro Burckardo alchimista maximo (8r); Illa duo habeo a magistro Johanne de Maguncia, magno practico (49v); Hoc habui a familiare domini de Lichtenberg, qui probanit et expertus est in scripto (27v); Sequuntur ea, que acquisini a carthusiensibus in Erfordia, que ipsi habuerunt ab vno experto viro phisico et cirurgico $\left(112^{\mathrm{v}}\right)$.

82 Vgl. 46r: Habeo ab hospite meo in Eystet; 48 : De granis juniperi ab hospite meo in Erfordia zw dem Stözel vtilissima sentencia (zu dem Erfurter Gasthof Zu dem Stötzel vgl. Bernhard Hartung, Die Häuser-Chronik der Stadt Erfurt, Erfurt 1861, S. 50 f.); 51v: Uor den bruch ein warhaftige kunst, die do zwgeht ane snidt, et fuit hospitis Zcum Swan Maguncie. 
Es fällt auf, dass sich unter den Rezeptautoren relativ viele Klosterangehörige befinden, Benediktinermönche aus Bamberg (151 r) und Scheyern (23r), Kartäuser aus Erfurt (112v), Freiburg i. Br. (27r), Regensburg (135r $)$, Seitz (100r) und Straßburg (1r), ein Zisterzienser aus Heisterbach (51v) sowie ein Deutschordensritter aus Frankfurt a. M. (54r). Mehrfach werden auch Rezepte von jüdischen Ärzten angeführt, so das Rezept eines Würzburger Juden für einen Wundtrank (Potus ad vulnera, quem judeus in Herbipoli fecit et est expertissimus, ideo secrete teneatur, $145^{v}$ ) oder ein Rezept bei Steinleiden, mit dem ein Jude den Mainzer Erzbischof Adolf von Nassau (Amtszeit 1461-1475) behandelt habe (Cum prima sequencium receptorum subuenit quidam judeus domino episcopo Maguntinensi, predecessori domini de Ysenberg, cui dedit 300 florenos, 49v-50r). 83

Die in der Sammlung am häufigsten genannte Stadt ist Straßburg. Möglicherweise hat der Schreiber hier seine Ausbildung als Wundarzt erhalten, auf jeden Fall bildete die Stadt eine wichtige Station, die prägend für seine spätere Berufstätigkeit war. Denn gleich am Anfang der Sammlung (1 ; vgl. Abb. 10) teilt er in einer Art Prolog mit, dass er dort von einem Kartäuser ein wertvolles Rezept für einen Wundbalsam erwarb, mit dem er später große medizinische Erfolge erzielte (darunter die bereits erwähnte Heilung einer Fistel in Leipzig):

Ad renerenciam domini nostri Ihesu Christi pandam aliquot secreta et paucis cognita humanisque corporibus necessaria, que acquisiui mibi a quodam Carthusiensi in Argentina, qui vocatur dominus Erbardus Knappe, cuius fama fulget per totam ciuitatem Argentinensem. Et illa, que michi tradidit, assumpsi ab eo in foro consciencie. Cum omni se dixit experienciam habere. Et cum hoc dixit, quod secreta illa, que michi traderet, vix pater filio tradere deberet. Quapropter diligenda et in scrinio pectoris recludenda. Balsamum enim artificialem vulnerum michi taxauit in consciencia sua pro 50 florenos, quos pocius, si in seculo esset, amittere quam alicui communicare. Per illum experiencias multas feci tam in vulneribus putridis quam recentibus. Et specialiter fistulam in Liepczk curaui, in qua plures manum apposuerunt, proficere autem illi non potuerunt.

Die einleitenden Sätze erwecken auf den ersten Blick den Eindruck, als ob es sich um einen Prolog zu der gesamten Sammlung handelte, in Wirklichkeit bezieht sich der Abschnitt jedoch nur auf die ersten drei, von dem Straßburger Kartäuser Erhardus Knappe stammenden (besonders wertvollen) Rezepte. Die Angabe, der Ruhm des Erhardus erstrahle per totam ciuitatem Argentinensem, impliziert, dass der Kompilator der Sammlung gut mit den Straßburger Verhältnissen vertraut war. In dieselbe Richtung weist auch die Nennung eines Straßburger Frühdruckers, dessen Name bisher nicht bekannt war: magister Bachstein, impressor librorum in Argentina (27r). Hinter diesem Namen dürfte sich wohl einer der

83 Daneben kommen noch zwei weitere Rezepte von jüdischen Gewährsmännern und Autoren vor: $50^{\mathrm{v}}$ ein Baderezept des Juden Joseph in Wiener Neustadt (Hanc artem habuit etiam Joseph judeus in Noua Cinitate, propter quam a Cesare et comitibus veneratur), $87^{\mathrm{r}}$ ein Tintenrezept eines Juden (De incausto vnius judei). 
anonymen Inkunabeldrucker verbergen, die in den 1470er- und frühen 1480erJahren in Straßburg wirksam waren. ${ }^{84}$

Ebenfalls auf einen Aufenthalt des Schreibers in Straßburg verweist der anscheinend nur in der Meißner Handschrift überlieferte gereimte deutsche Pesttraktat (Pestregimen), der sich auf Blatt $75^{\mathrm{v}}-81^{\mathrm{v}}$ findet. ${ }^{85}$ Die Abfassung dieses ca. 250 Verse umfassenden, auf recht bescheidenem Niveau gereimten Gedichts wurde wohl, wie aus einer Stelle im Prolog hervorgeht, durch eine in der Gegend von Straßburg und Mainz grassierende Pestepidemie veranlasst: $N u$ vindet man manchen jungen ond alden, / die nicht wissen, wie sie ordenung sullen halden / ond sich sullen bebüten zw dießer frist / vor dem kurczen sterben, das do ist, / ich meyne vor der pestilencz, / die do ist vmb Straßburg ond vmb Meincze (76 $\left.{ }^{\mathrm{r}-\mathrm{v}}\right)$. Eine Entstehung in Straßburg ist sehr viel wahrscheinlicher als in Mainz, denn die Reimsprache des Werks ist elsässisch. ${ }^{86}$ Am Schluss des Gedichts nennt der Autor die Initialen seines Namens: C. K. (als ich C. K. in den buchern bab geleßen, 81 ${ }^{\mathrm{r}}$ ).

Die ganze Sammlung macht vom Äußeren her einen sehr wohlgeordneten und einheitlichen Eindruck: Sie ist von einer einzigen Hand in einem Arbeitsgang geschrieben, jedes Rezept beginnt gewöhnlich mit einer zwei- oder dreizeiligen roten Lombarde (einer unverzierten Initiale) und einer durch größeren Schriftgrad hervorgehobenen Überschrift (vgl. Abb. 11), es handelt sich offenbar um die Reinschrift einer Rezeptsammlung.

Im Gegensatz dazu ist der Inhalt sehr heterogen und weist kaum irgendeine erkennbare Ordnung auf. Den inhaltlichen Kern bilden medizinische, überwiegend chirurgische Rezepte. Nur relativ selten sind diese zu kleineren oder größeren Gruppen zusammengefasst. So sind in einigen Fällen mehrere Rezepte zusammengestellt, die von einem Gewährsmann stammen (10r-19r Petrus Wertwein von Coburg, 19v Michael von Reichenau, 145v-148v Meißner Domherr Hildebrand [s. o.], 151 ${ }^{\mathrm{r}}-153^{\mathrm{v}}$ Georg von Schauenberg, Benediktiner aus Bamberg, 153 $3^{\mathrm{v}}-155^{\mathrm{r}}$ Magister Tywaldus von Pavia, 155 ${ }^{\mathrm{r}}-156^{\mathrm{r}}$ Magister Johannes Unbehauen). Etwas häufiger sind Abschnitte, die eine Anzahl von Rezepten zu einer bestimmten Krankheit oder einem bestimmten Anwendungsgebiet vereinigen (z. B. $7^{r}-8^{r}$ und 37v-39r Rezepte zur Gynäkologie, 43r-v zur Behandlung von Wurmleiden, 57r-61 r

$84 \mathrm{Zu}$ den Straßburger Druckern vgl. Ferdinand Geldner, Die deutschen Inkunabeldrucker. Ein Handbuch der deutschen Buchdrucker des XV. Jahrhunderts nach Druckorten, Stuttgart 1968, S. 55-86.

85 Das Gedicht ist überschrieben Regimen pestilencie, sein Incipit lautet: Es nv gewonlich ist, I das ein iclich mensch begeret lange frist / zw leben, als Pawlus ad Corintheos schriebet. $\mathrm{Zu}$ den bisher bekannten gereimten deutschen Pestregimina des Spätmittelalters (u. a. von Hans Andree, Hans Folz und Heinrich Laufenberg) vgl. die Literaturhinweise in meiner Handschriftenbeschreibung (wie Anm. 75).

86 Reime wie gebraten: gesoten, gan: schon und rat: god lassen auf eine Verdumpfung von $\hat{a} \mathrm{zu} o$ in der Sprache des Autors schließen (d. h. auf eine Aussprache gebroten, gon, rot); es handelt sich dabei um eines der hervorstechendsten Merkmale der Schreibsprache des Elsass; vgl. Wolfgang Kleiber/Konrad Kunze/Heinrich Löffler, Historischer Südwestdeutscher Sprachatlas. Aufgrund von Urbaren des 13. bis 15. Jahrhunderts, Bd. II, Bern/München 1979, Karten 38-41. 


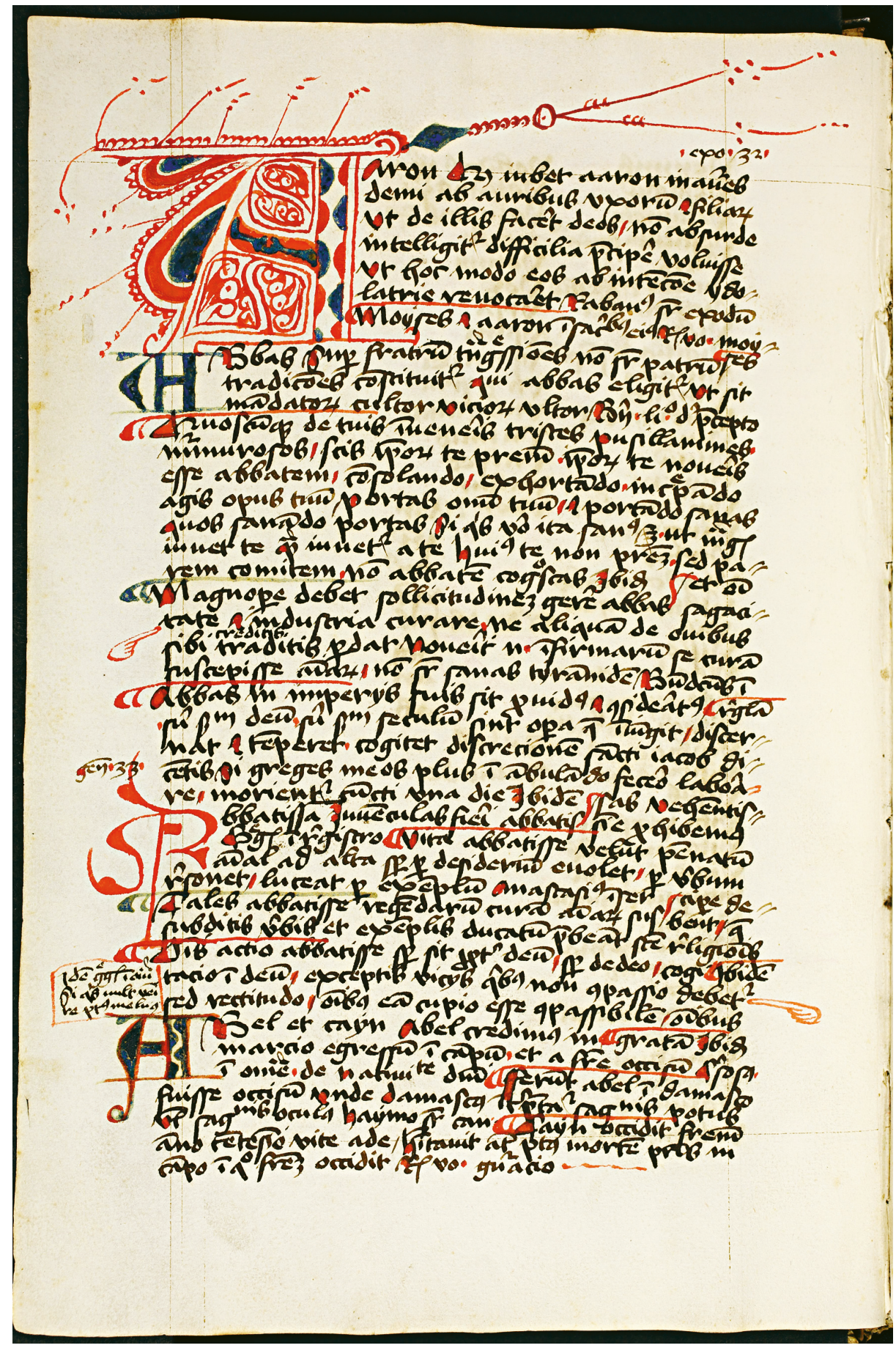

Abb. 1: Beginn der Tabula originalium des Franziskaners Johannes Erfordensis. 


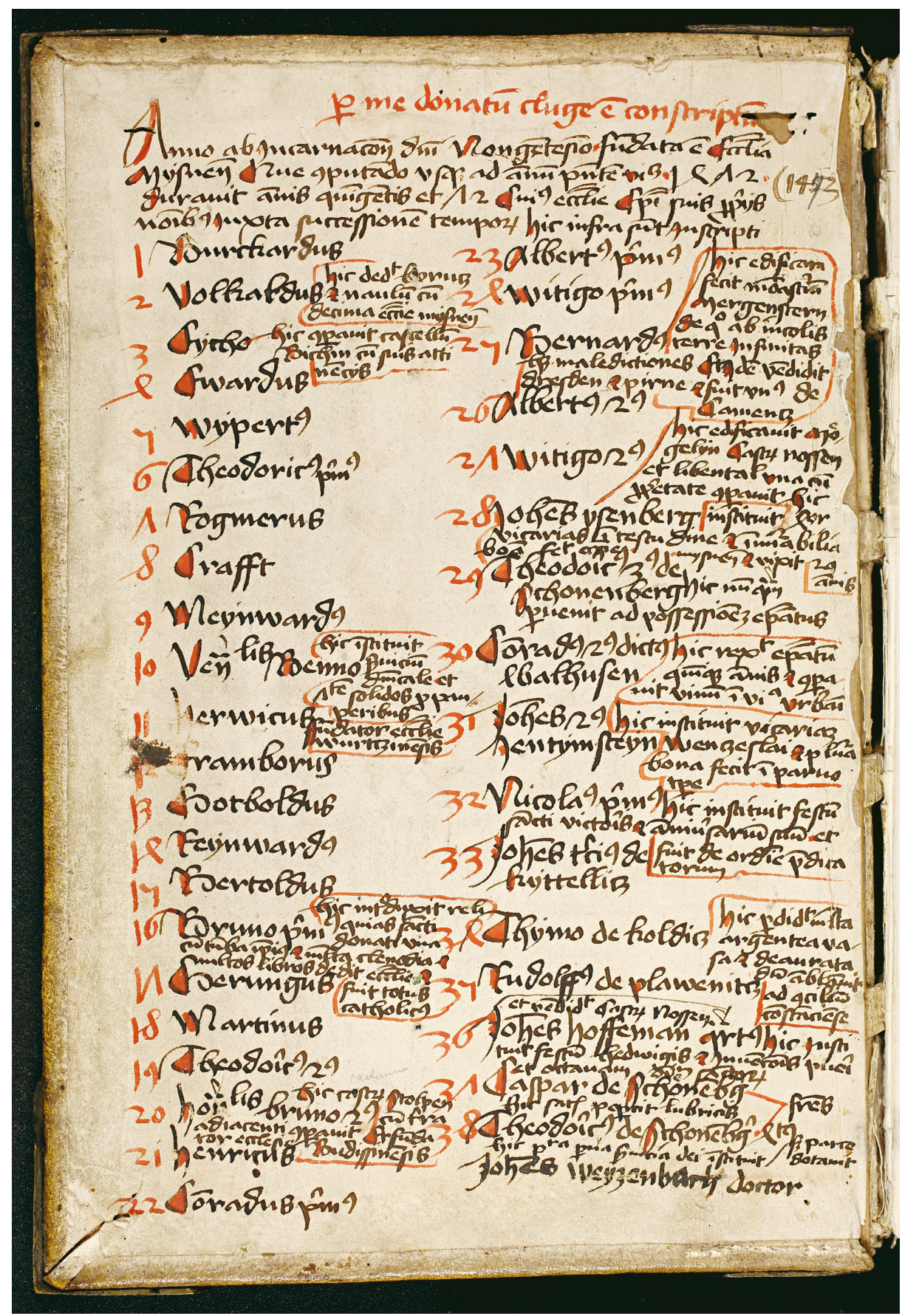

Abb. 2: Liste der Meißner Bischöfe, geschrieben 1472 von Donatus Cluge. 


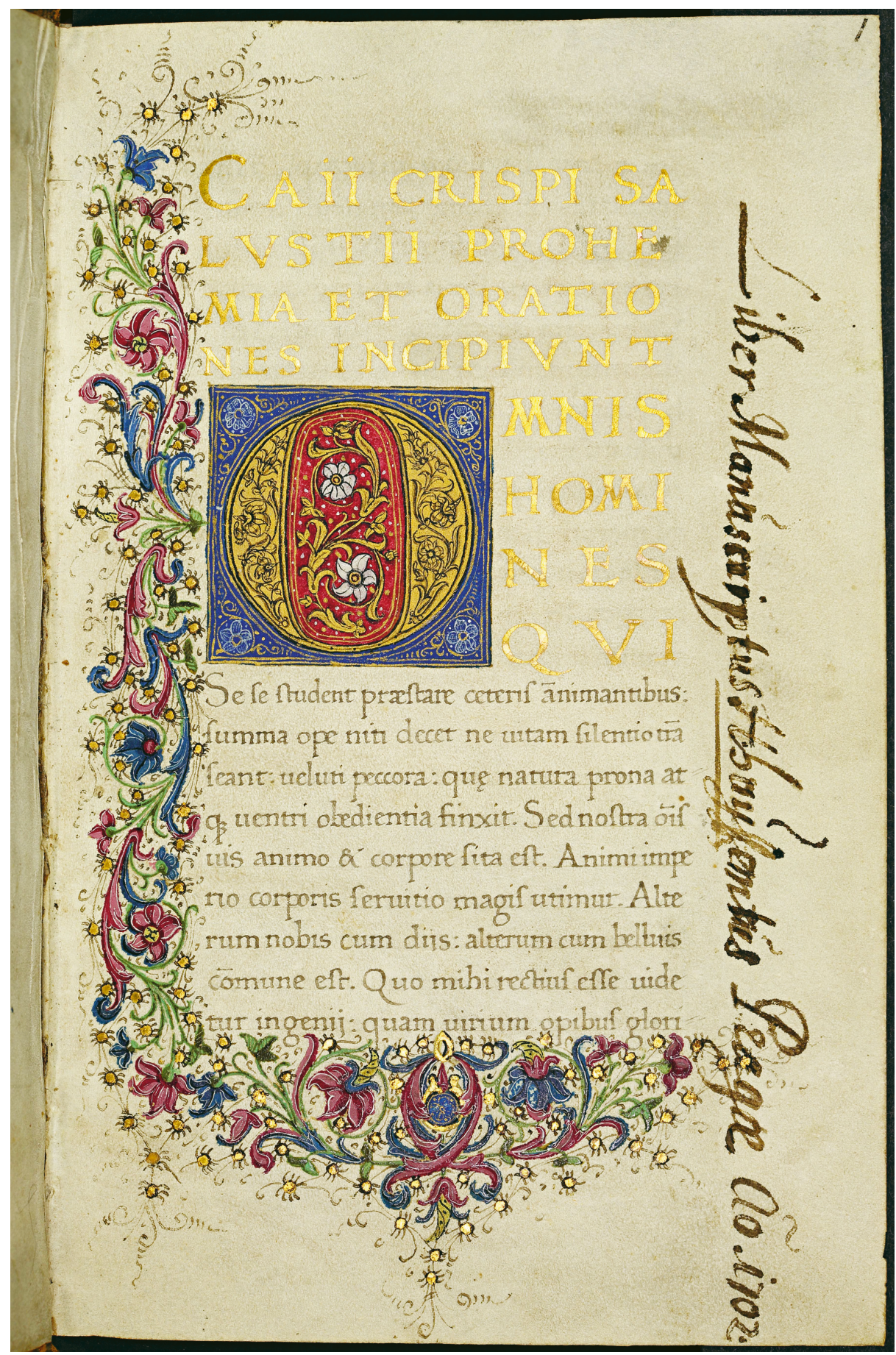

Abb. 3: Zierseite einer rhetorischen Sammelhandschrift, Florenz (?), um 1480/90. 


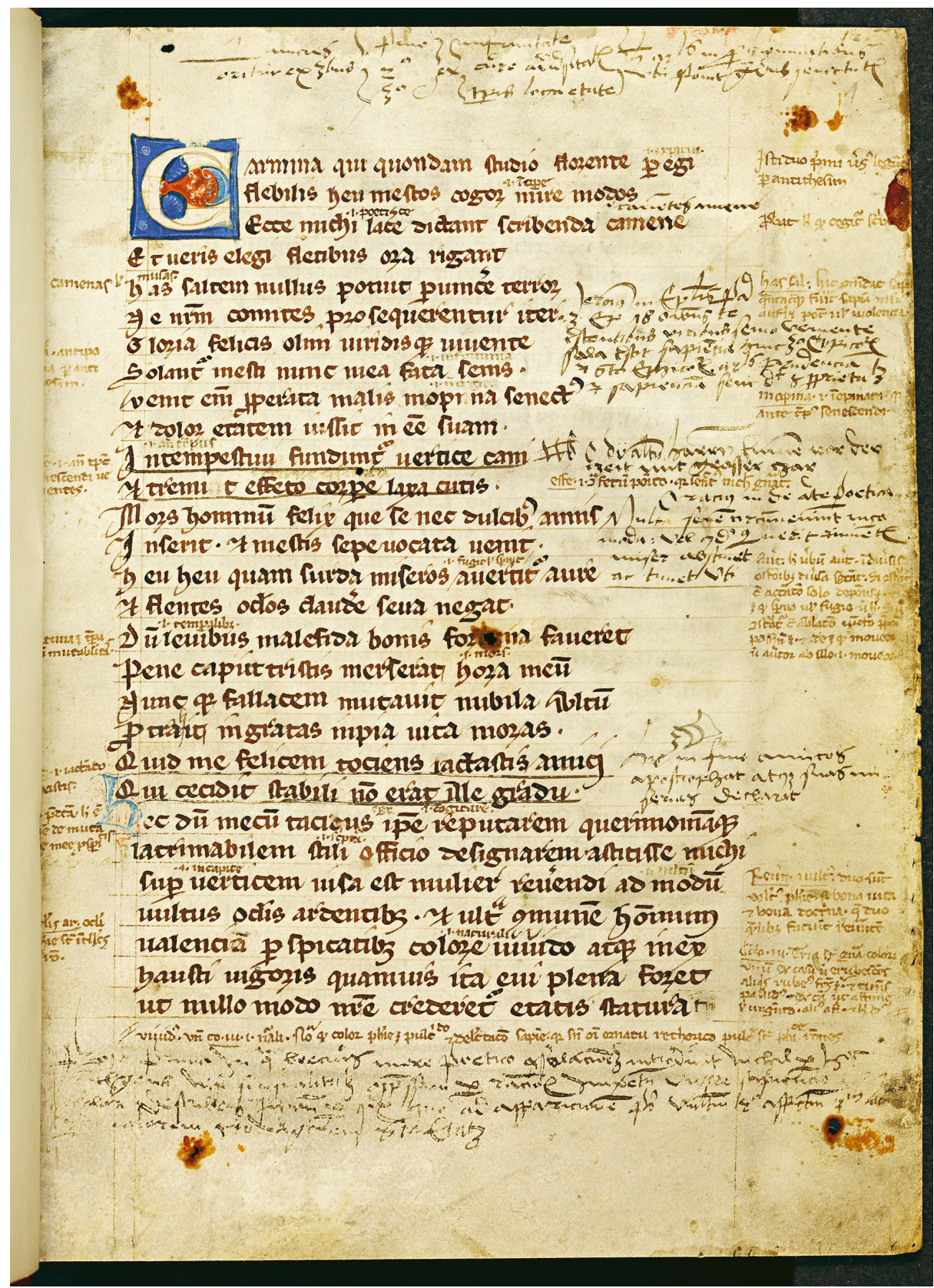

Abb. 4: Textbeginn von Boethius: Consolatio philosophiae, Südfrankreich oder Oberitalien, letztes Viertel des 13. Jabrhunderts. 


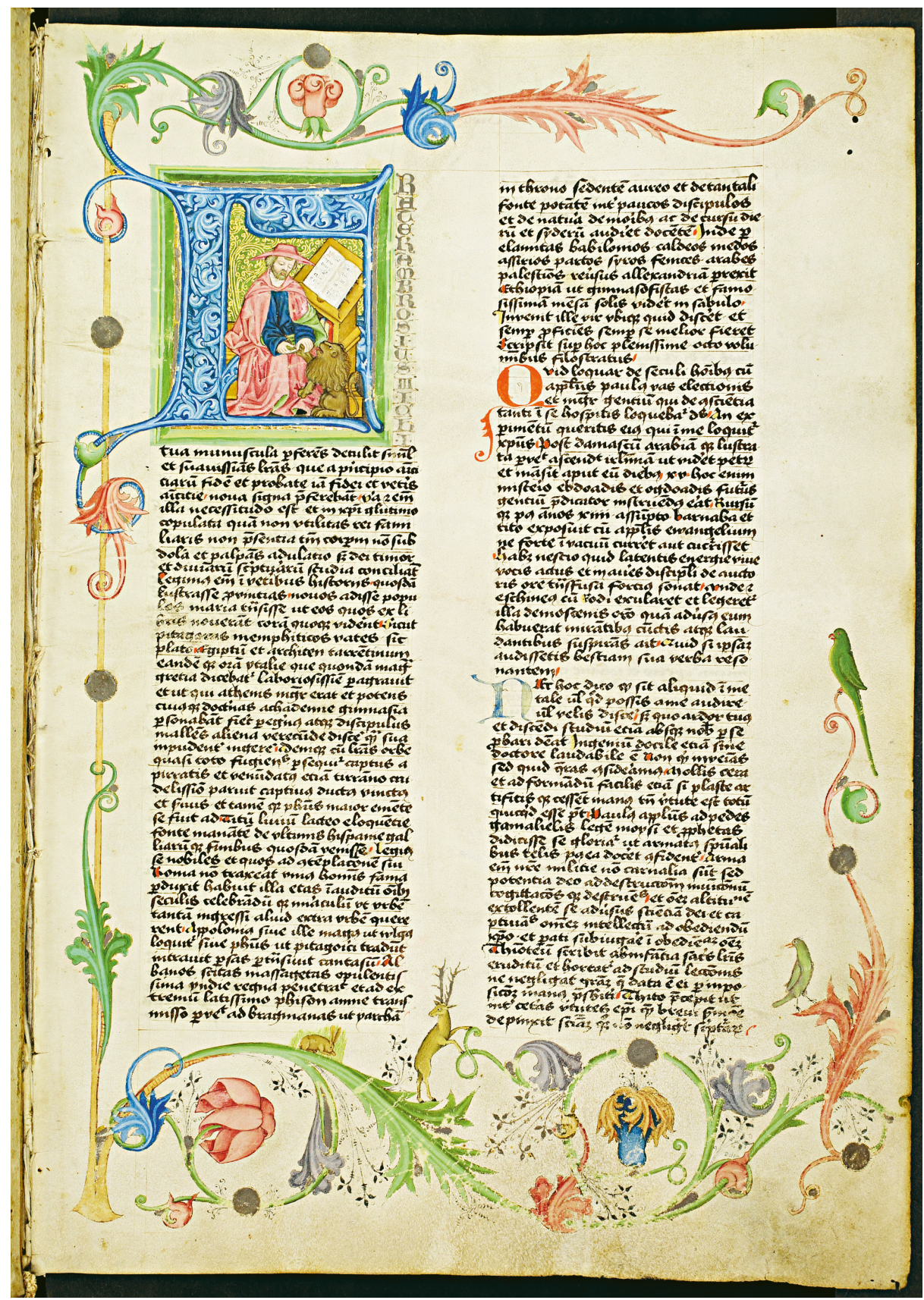

Abb. 5: Beginn des Alten Testaments mit Autorenbild des Hl. Hieronymus in der von Jan Mikuš ausgestatteten Bibel. 


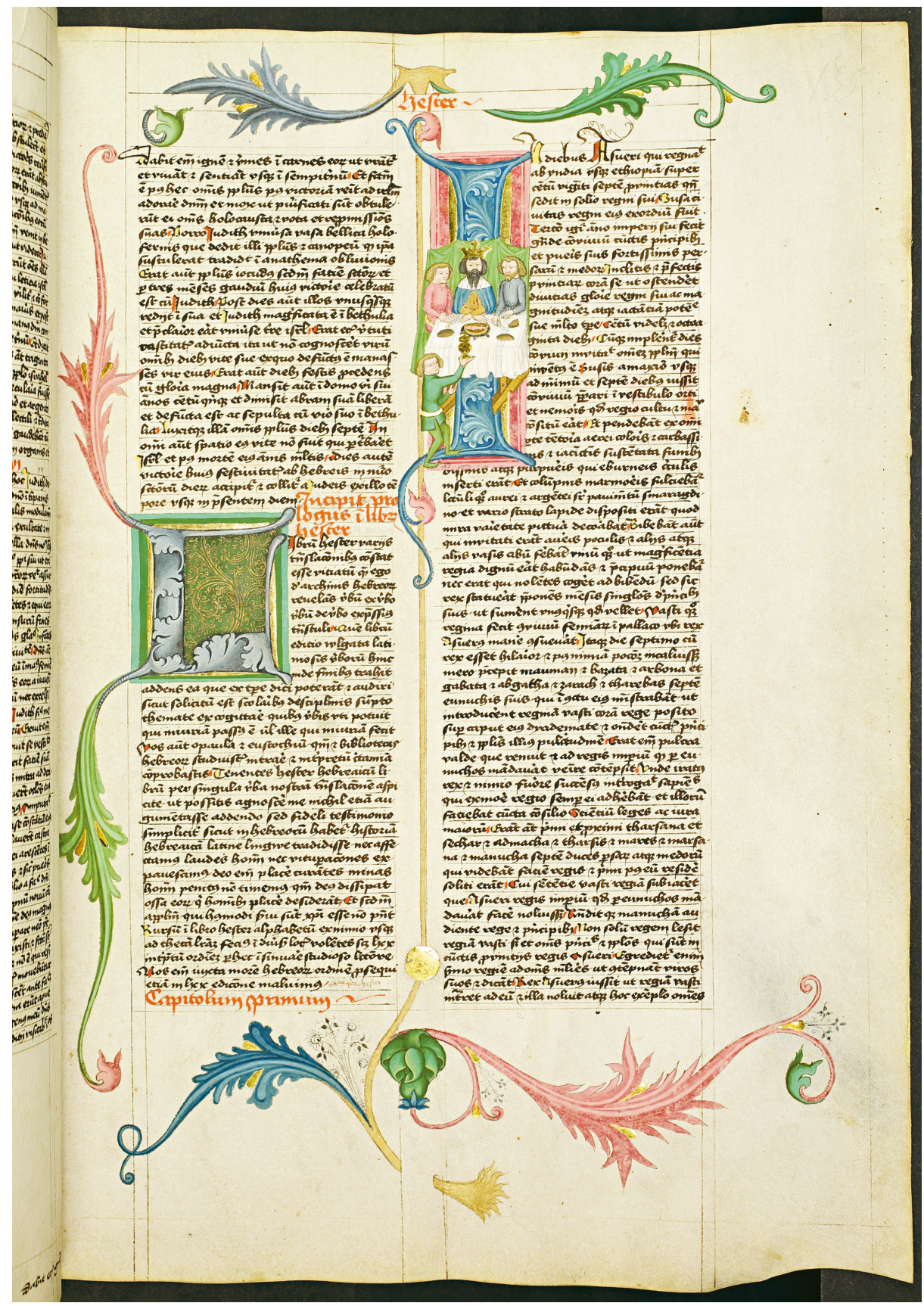

Abb. 6: Beginn des Buches Esther in der von Jan Mikušausgestatteten Bibel. 


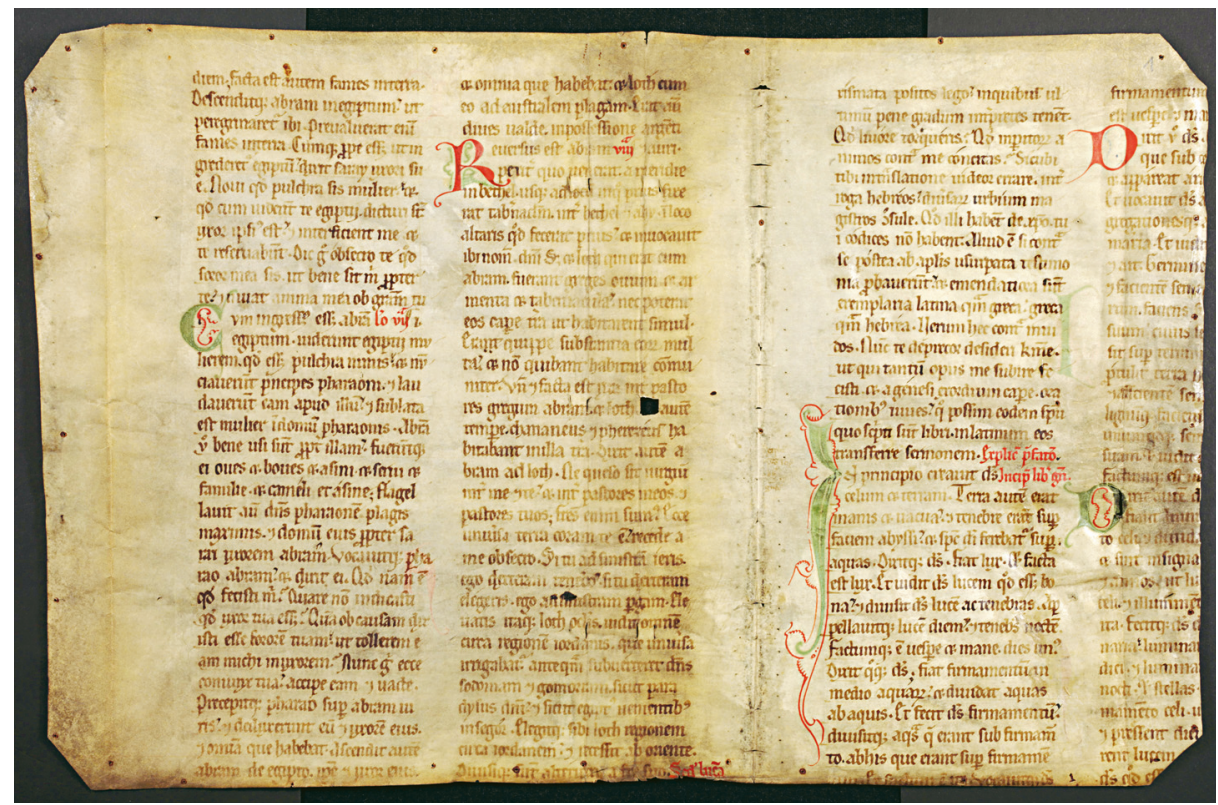

Abb. 7: Fragment eines Zisterzienser-Lektionars.

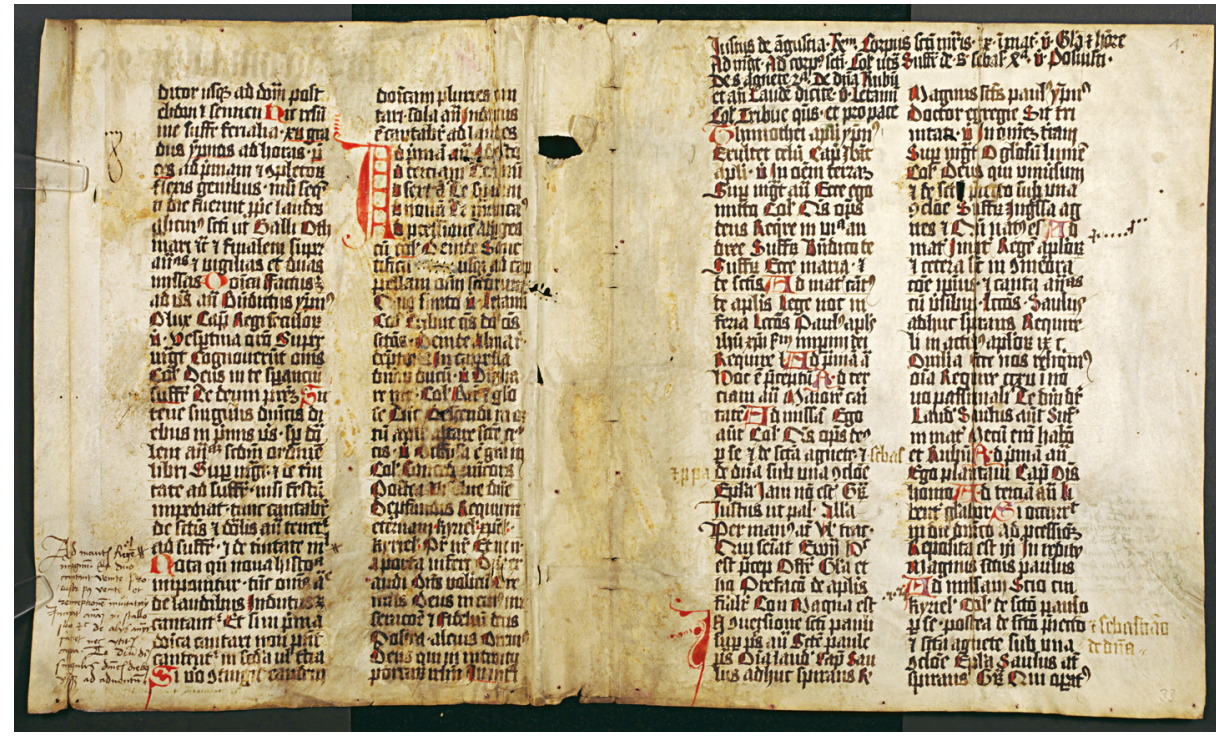

Abb. 8: Fragment eines Liber ordinarius des Meißner Doms. 


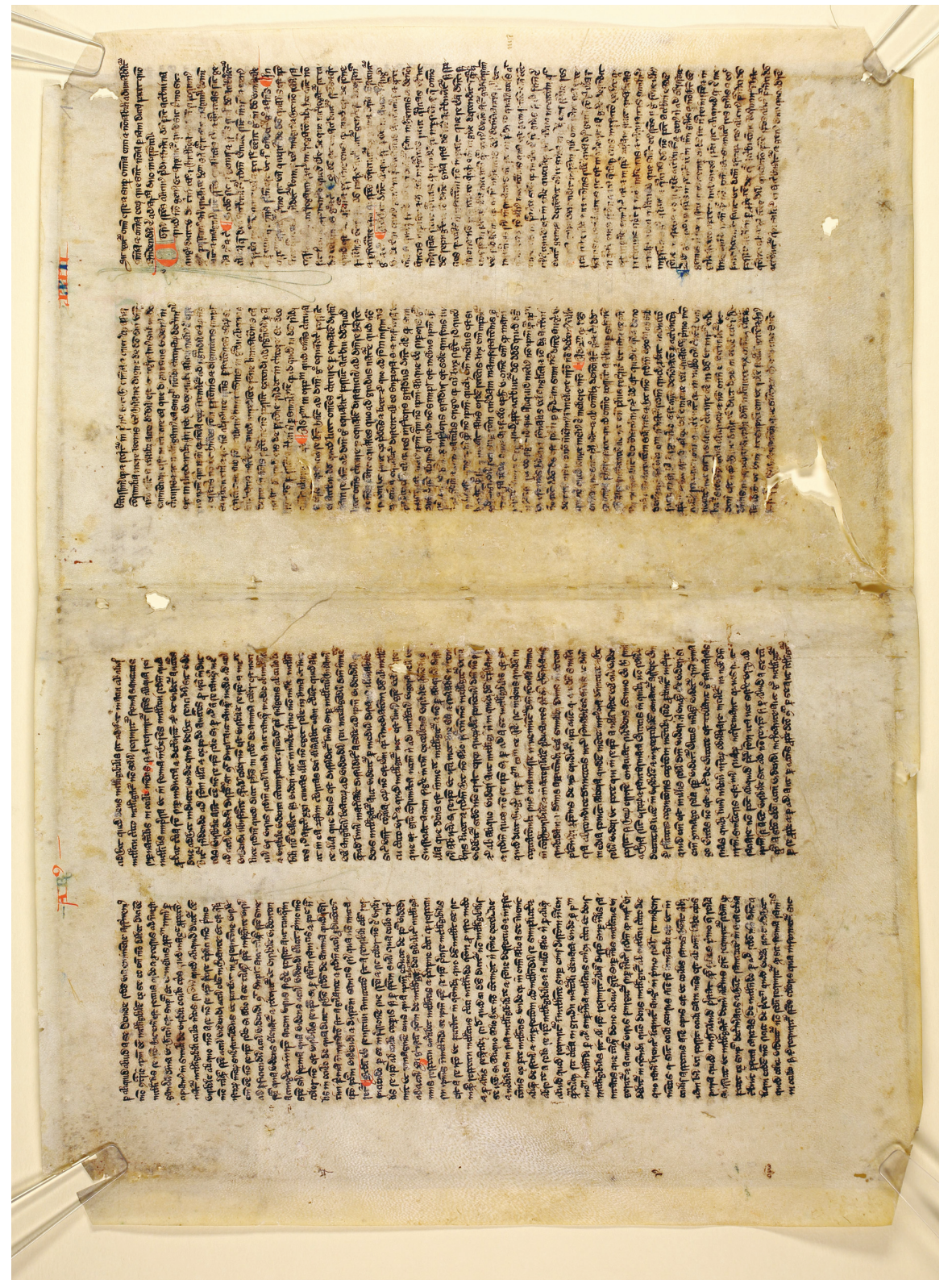

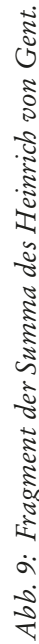




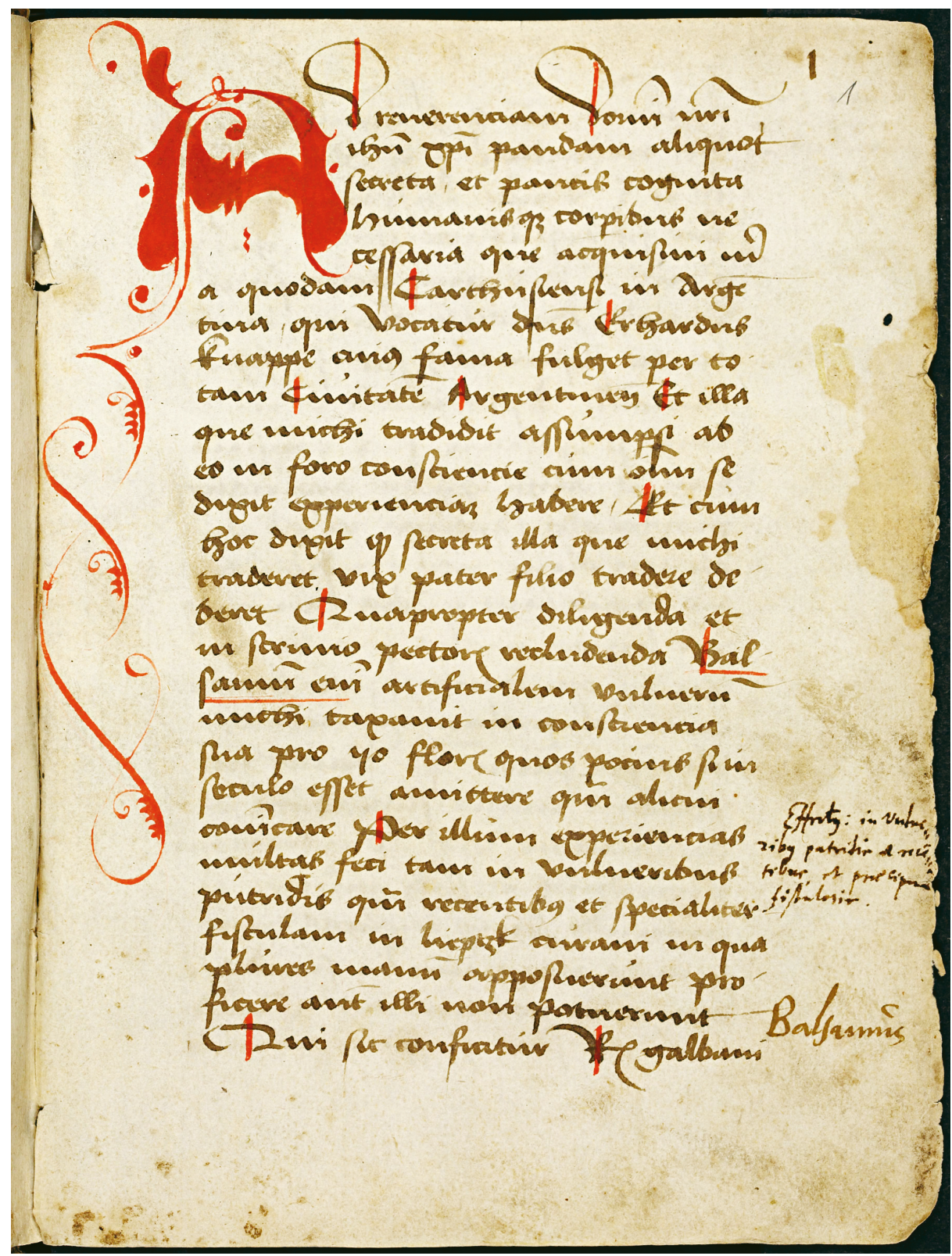

Abb. 10: Beginn der Rezeptsammlung eines Wundarztes, Meißen oder Leipzig, um 1485. 


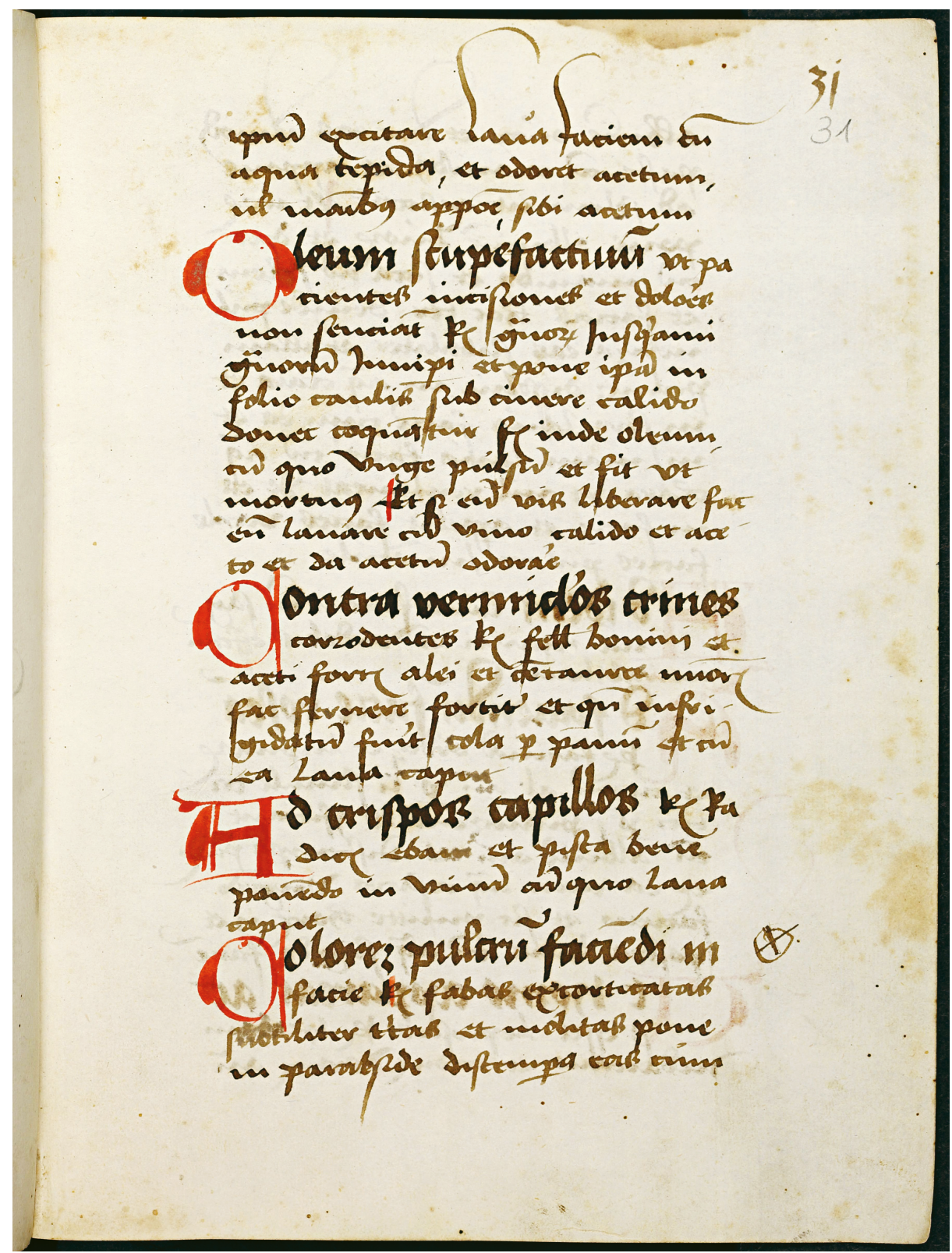

Abb. 11: Rezept für Betäubungsmittel, Schönheitsrezepte. 
요

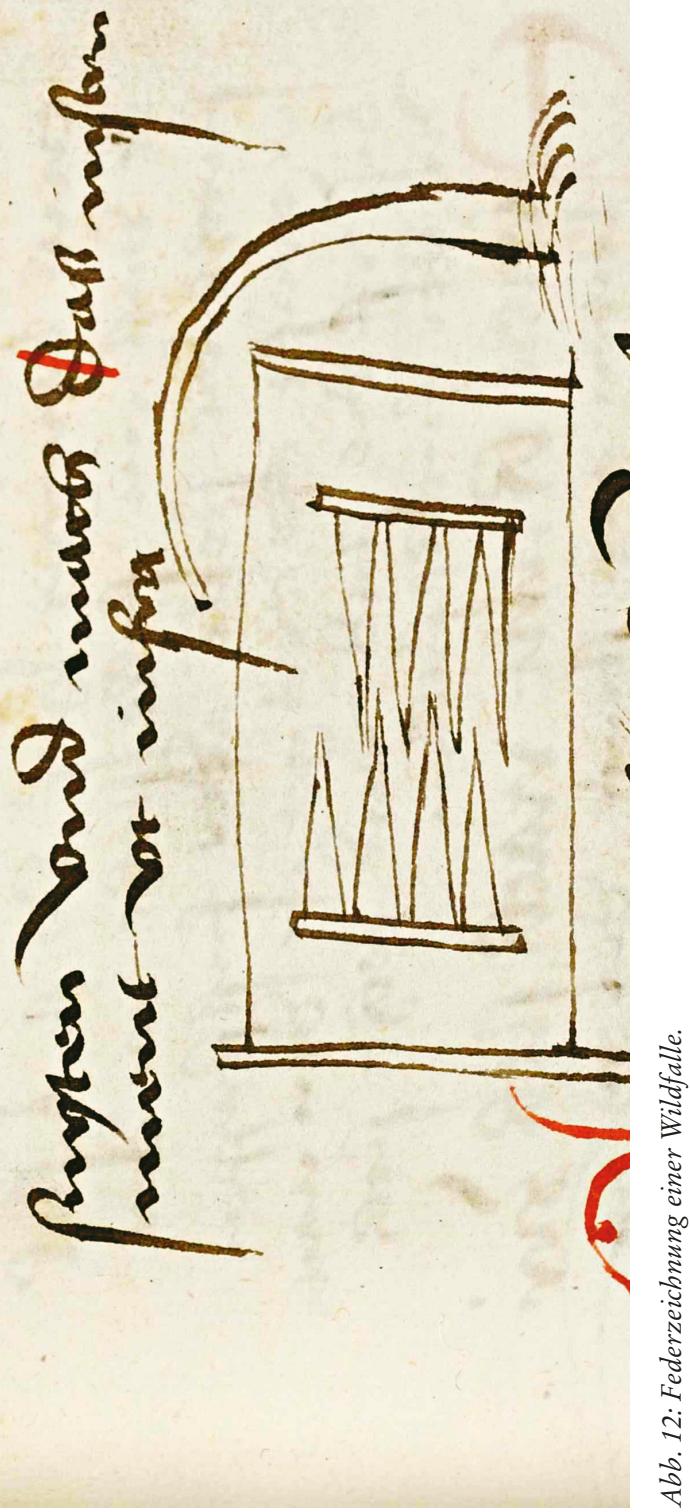


Rezepte gegen die Pest, 70r-v gegen Geschwulste, 100r-101 ${ }^{\mathrm{r}}$ gegen Augenkrankheiten, $110^{\mathrm{v}}-111^{\mathrm{r}}$ gegen Steinleiden, $142^{\mathrm{r}}-143^{\mathrm{r}}$ gegen Hautkrankheiten).

Zwischen den humanmedizinischen Rezepten, die insgesamt den weitaus größten Raum in der Sammlung einnehmen, finden sich immer wieder in bunter Aneinanderreihung Rezepte $\mathrm{zu}$ den verschiedenartigsten Themen eingestreut: Rezepte für Schönheitsmittel (31 r (vgl. Abb. 11), 44r $)$, Haarfärbemittel $\left(21^{\mathrm{r}}, 83^{\mathrm{r}}\right)$ und Mittel zur Fleckentfernung (22r); Weinrezepte (66 $\left.{ }^{\mathrm{r}}, 74^{\mathrm{r}}, 145^{\mathrm{r}}\right)$; technische und chemische Rezepte, z. B. zum Vergolden von Metallen $\left(6^{r}, 64 r\right)$, zum Herstellen von Gipsabgüssen $\left(6^{\mathrm{v}}\right)$, zur Herstellung von Tinte (85v) oder zur Scheidung von Metallen (127r); veterinärmedizinische Rezepte, vor allem für Rossarzneien (44r, 46 $\left.{ }^{\mathrm{v}}, 101^{\mathrm{r}}, 139^{\mathrm{r}}\right)$; alchemistische Rezepte (48r , 100r, 144r); schließlich auch Anleitungen für Fischfang $\left(42^{\mathrm{v}}, 137^{\mathrm{r}}-138^{\mathrm{v}}\right)$ und Jagd $\left(45^{\mathrm{r}}\right.$, 133 v-137r, $138^{\mathrm{v}}$, mit einer Federzeichnung einer Wildfalle $135^{\mathrm{r}}$ (vgl. Abb. 12)).

Interessant ist die Sprache der Handschrift, die fortwährend zwischen Latein und Deutsch wechselt. Insgesamt überwiegen die lateinischen Teile. Die medizinischen Rezepte sind meist lateinisch, die nichtmedizinischen meist deutsch. In vielen Stücken werden beide Sprachen nebeneinander benutzt. Am häufigsten ist der Fall, dass die Überschrift bzw. die Einleitung lateinisch und der Haupttext deutsch oder umgekehrt die Überschrift deutsch und der Haupttext lateinisch ist. In einer Reihe von Rezepten wechseln sich längere deutsche und lateinische Passagen ab, in einigen Fällen sind einzelne lateinische Ausdrücke in ansonsten rein deutschen Text eingefügt. Darüber hinaus gibt es auch Rezepte, in denen eine regelrechte lateinisch-deutsche Mischsprache gebraucht wird; ein instruktives Beispiel dafür ist das Rezept Bl. 23 ${ }^{\mathrm{r}}-26^{\mathrm{r}}$, in dem ein ständiger Wechsel zwischen beiden Sprachen stattfindet; vgl. zum Beispiel eine Stelle Bl. 24v: In quarta distillacione efficitur aqua virtuosior vnd vorbrent tuch, holcz vnd alles anders, was man dor in thud, et est tante virtutis, ut omnes vires hominis confortet [...], auch nymt das wasser an sich virtutes omnium herbarum.

Der Schreiber hat der ungeordneten Rezeptsammlung ein alphabetisches Sachregister $\left(158^{r}-163^{r}\right)$ angefügt und diese damit als Nachschlagewerk nutzbar gemacht. Mit Sicherheit war die Sammlung nicht nur für seinen eigenen Gebrauch bestimmt, sondern auch (und vor allem) für Schüler oder Nachkommen, die sich ebenfalls als Wundarzt betätigten. Mehrfach spricht er in der ersten Person und kündigt an, im Folgenden geheimes medizinisches Wissen auszubreiten: Pandam aliquot secreta et paucis cognita (1r), Pandam bic magna secreta (100r), Nunc pandam vobis (88) $)$. An zahlreichen Stellen bezeichnet er den Inhalt von Rezepten als geheim und wenigen bekannt: Unum magnum secretum et paucis cognitum pro mulieribus in partu eam facilitando $(7 \mathrm{r})$; De [...] magno secreto contra epidimiam (10r); Secretum maximum, in quo magni philosophi maxime confidebant $\left(23^{\mathrm{r}-\mathrm{v}}\right)$; illud est secretum et nemini voluit communicare (65) $)$; Medicina mirabilis et secreta $(174 \mathrm{r})$.

Immer wieder betont er den großen Nutzen von einzelnen Rezepten (z. B. 48 De granis juniperi [...] vtilissima sentencia, $100 \mathrm{v}$ aqua vtilis pro oculis curandis); dies 
gelte besonders in materieller Hinsicht; so versichert er, dass der Wert eines Wundbalsam-Rezepts auf 50 Gulden geschätzt werde (1r), und teilt ein Rezept mit, dessen Preis er sogar auf 100 Gulden schätzt (51v; vgl. auch $27^{\mathrm{r}}$, 49v und 85v). Die genannten Summen implizieren, dass die betreffenden Rezepte eine zuverlässige Einnahmequelle bei der Ausübung des ärztlichen Berufs bildeten, explizit hingewiesen wird darauf beispielsweise bei einem Rezept eines Mainzer Kanonikers, mit dessen Hilfe dieser in Pestzeiten viel Geld verdient habe: Aurum vite, quod [...] valet contra pestilenciam et febres, cum quo magister Nicolaus, Maguntinensis canonicus, capellanus quondam domini palatini, magnam peccuniam tempore pestis acquisinit (17v), oder bei einem chirurgischen Rezept nor alde vnd new scheden, für das ein Straßburger Drucker wolt geben 36 florenos, ex qua quis posset habere nutrimentum (27r).

Zur Begründung, warum ein bestimmtes Rezept besonders wert- und wirkungsvoll sei, kann auch der Hinweis dienen, dass dieses bei prominenten Patienten angewendet worden sei bzw. von einem Arzt stamme, der im Dienst eines hohen Adligen stand: Von einem Steinrezept heißt es, dass ein namentlich nicht genannter Magister damit dem Herzog von Bayern geholfen habe und dass es sich auch bei verschiedenen anderen Patienten bewährt habe, darunter dem Grafen von Katzenelnbogen (49v-50r). Bei einem Rezept gegen den Bruch wird ebenfalls versichert, dass es bei einem Herzog von Bayern erfolgreich gewesen sei (53 $3^{\mathrm{r}-\mathrm{v}}$ fur den bruch, expertum in vno duce Bauarie). Bl. 65r findet sich ein Rezept für ein electuarium (Latwerge), das von Doctor Münsinger (wohl Albrecht Münsinger, 1458-1509), Arzt in Diensten der pfälzischen Kurfürsten, stammt. ${ }^{87} \mathrm{Zu}$ verweisen ist ferner auf den oben bereits vorgestellten Meißner Domherren Hildebrand, der Leibarzt der Herzöge von Sachsen war und als Gewährsmann für eine Serie von insgesamt 21 Rezepten angeführt wird (145v $\left.-148^{\mathrm{v}}\right)$.

Bei der Sammlung handelt es sich um ein stark praxisbezogenes Manual für einen Wundarzt. Der Bezug zur praktischen Arbeit äußert sich auf der einen Seite darin, dass bei zahlreichen Rezepten hervorgehoben wird, dass sie vielfach erprobt worden seien, immer wieder wird dabei das Adjektiv expertus (z. B. 104v composicio optima per me sepius experta, $61^{\mathrm{r}}$ expertus sum, $112^{\mathrm{v}}$ sunt vera et experta) oder das Verb probare $\left(88^{\mathrm{r}}\right.$ velut ipse probani, $151^{\mathrm{v}}$ aqua bona et probata) verwendet. Auf der anderen Seite zeigt sich dies darin, dass - wie schon angedeutet - gelehrtes, theoretisches Wissen in Form von bekannten gelehrten Texten der Universitätsmedizin so gut wie gar nicht in der Sammlung auftaucht; die zahlreichen Rezeptautoren sind mehrheitlich praktische Ärzte, nur wenige lassen sich in Universitätsmatrikeln nachweisen. An mehreren Stellen wird der Gegensatz zu dem

$87 \mathrm{Zu}$ diesem vgl. Werner Friedrich Kümmel, Albrecht Münsinger, in: Verfasserlexikon (wie Anm. 3), Bd. 6 (1987), Sp. 780-783. Aus chronologischen Gründen weniger wahrscheinlich ist eine Identifizierung mit dessen Vater Heinrich (um 1397-1476), der ebenfalls Leibarzt der pfälzischen Kurfürsten war; vgl. Gundolf KeIL, Heinrich Münsinger, in: ebd., Sp. 783-790. 
gelehrten Arzt angedeutet (vgl. $171^{\mathrm{r}}$ quod latini dicunt, $172^{\mathrm{v}}$ secundum medicos). ${ }^{88}$ Dass die Sammlung ganz auf die Verwendung in der wundärztlichen Praxis ausgerichtet ist, wird besonders deutlich an einem Rezept für ein Betäubungsmittel bei Operationen, das überschrieben ist: oleum stupefactiuum, vt pacientes incisiones et dolores non senciant (31 ${ }^{\mathrm{r}}$, vgl. Abb. 11).

Dass die Sammlung tatsächlich, wie von ihrem Kompilator beabsichtigt, von Späteren benutzt wurde, zeigt der Umstand, dass sie gegen Ende des 15. Jahrhunderts von mehreren Händen ergänzt wurde, die zahlreiche, ausschließlich medizinische Rezepte eintrugen $\left(163^{\mathrm{v}}-189^{\mathrm{r}}\right)$. Weitere Nachträge wurden noch in der 2. Hälfte des 16. Jahrhunderts angefügt (189r-190v). Unter den Nachträgen befinden sich drei bisher unbekannte Rezepte des renommierten Freiberger Stadtarztes Nikolaus Münzmeister (185'r $\left.188^{\mathrm{v}}-189^{\mathrm{r}}\right)$, von denen eines für den Naumburger Bischof bestimmt war, sowie ein Pestrezept (puluis contra pestem) eines nicht identifizierten Nicolaus ...lschman (Anfangsbuchstaben des Namens durch Tintenklecks verdeckt), der als informator principum Saxonie bezeichnet wird.

Die Handschrift H 6 ist vor allem wichtig als Zeugnis eines bislang unbekannten (anonymen) Meißner Stadtarztes, der in ihr eine umfangreiche Sammlung von Rezepten zusammenstellte, die er seinen Nachfolgern als Vermächtnis hinterließ. Im Unterschied zu durchschnittlichen mittelalterlichen Arzneibüchern und medizinischen Manuales, die zumeist in großen Teilen aus bekannten Quellen exzerpiert sind, lassen sich in ihr kaum irgendwelche schriftlichen Vorlagen nachweisen. Das Besondere an der Sammlung ist, dass sie ganz aus der wundärztlichen Praxis erwachsen ist und vornehmlich Rezepte enthält, die entweder von dem Meißner Wundarzt selbst oder von Kollegen in der praktischen Arbeit erprobt worden waren.

\section{Fazit}

Die wissenschaftliche Tiefenerschließung der Handschriften und Fragmente des Meißner Stadtarchivs führte zu vielen Neuentdeckungen, die einerseits die Lokalgeschichte Meißens betreffen, andererseits über die Stadt hinausweisen, etwa in den böhmischen, italienischen und französisch-englischen Raum. Noch in vielen städtischen und kirchlichen Archiven „schlummern“ solche unbearbeiteten Bestände, sowohl in Form von einzelnen mittelalterlichen Buchhandschriften als auch (in großem und noch nicht abzuschätzendem Umfang) in Form von Aktenoder Bucheinbänden aus makulierten Handschriften. Die Projektergebnisse der Kleinsammlungsprojekte am Leipziger Handschriftenzentrum zeigen, dass sich die Erschließung dieser Bestände lohnt und durch sie neues Material sowohl für die landesgeschichtliche Forschung als auch für andere Forschungsbereiche zur Verfügung gestellt wird.

88 Die beiden Stellen stehen zwar in den Nachträgen, die gleiche Haltung gilt jedoch auch für den Haupttext. 